\title{
Review
}

Jiafeng Lu, Fan Shi, Jiangtao Xu, Linghao Meng, Longkun Zhang, Peikang Cheng, Xuan Zhou, Fufei Pang and Xianglong Zeng*

\section{Recent progress of dynamic mode manipulation via acousto-optic interactions in few-mode fiber lasers: mechanism, device and applications}

https://doi.org/10.1515/nanoph-2020-0461

Received August 7, 2020; accepted November 9, 2020; published online November 24, 2020

\begin{abstract}
The burgeoning advances of spatial mode conversion in few-mode fibers emerge as the investigative hotspot in novel structured light manipulation, in that, high-order modes possess a novel fundamental signature of various intensity profiles and unique polarization distributions, especially orbital angular momentum modes carrying with phase singularity and spiral wave front. Thus, control of spatial mode generation becomes a crucial technique especially in fiber optics, which has been exploited to high capacity space division multiplexing. The acousto-optic interactions in few-mode fibers provide a potential solution to tackle the bottleneck of traditional spatial mode conversion devices. Acousto-optic mode conversion controlled by microwave signals brings tremendous new opportunities in spatial mode generation with fast mode tuning and dynamic switching capabilities. Besides, dynamic mode switching induced by acoustooptic effects contributes an energy modulation inside a laser cavity through nonlinear effects of multi-mode interaction, competition, which endows the fiber laser
\end{abstract}

\footnotetext{
*Corresponding author: Xianglong Zeng, The Key Lab of Specialty Fiber Optics and Optical Access Network, Joint International Research Laboratory of Specialty Fiber Optics and Advanced Communication, Shanghai University, Shanghai 200444, China,

E-mail: zenglong@shu.edu.cn

Jiafeng Lu, Fan Shi, Jiangtao Xu, Linghao Meng, Longkun Zhang, Peikang Cheng, Xuan Zhou and Fufei Pang, The Key Lab of Specialty Fiber Optics and Optical Access Network, Joint International Research Laboratory of Specialty Fiber Optics and Advanced Communication, Shanghai University, Shanghai 200444, China,

E-mail: jiafenglu@shu.edu.cn (J. Lu), stone91@shu.edu.cn (F. Shi), tsyjxjt@shu.edu.cn (J. Xu), Dreamhao@shu.edu.cn (L. Meng), zlk18724776@shu.edu.cn (L. Zhang),cpk123456@shu.edu.cn (P. Cheng), zhouxuan123@shu.edu.cn (X.Zhou), ffpang@shu.edu.cn (F. Pang). https://orcid.org/0000-0002-2691-6688 (J. Lu). https:// orcid.org/0000-0002-7106-4584 (F. Pang)
}

with new functions and leads to the exploration of new physical mechanism. In this review, we present the recent advances of controlling mode switch and generation employing acousto-optic interactions in few-mode fibers, which includes acousto-optic mechanisms, optical field manipulating devices and novel applications of spatial mode control especially in high-order mode fiber lasers.

Keywords: acousto-optic interactions; dynamic mode manipulation; fiber laser; orbital angular momentum; spatial mode conversion.

\section{Introduction}

Spatial modes composed by cylindrical vector modes (CVMs), linear polarization (LP) modes and optical vortex modes exhibit special distributions of electrical fields existing stably in optical fibers [1-3]. Given the increasing growth of network traffic, the manifestation of these spatial modes could imply the future communication technology through spatial division multiplexing (SDM) [4-8]. Meanwhile, light beam shaping in spatial domain incorporating fiber laser technique has found diverse applications in optical tweezers [9-11], structured light imaging [12-16], quantum information science [17-20], laser manufacture [21-24] and optical communication [25-28].

Dynamic mode manipulation should be considered during generating spatial high-order modes (HOMs) directly, which can greatly improve the practical utilities of these light sources. Static methods of generating spatial modes in optical fibers can be mainly classified into four categories: mode selective couplers (MSCs) [29-31], photonic lanterns [32-34], long-period gratings (LPGs) [35-38] and fiber Bragg gratings (FBGs) [39-41]. MSC employs tapering fused fibers to demonstrate mode coupling via evanescent field interactions. Combining a single-mode fiber (SMF) and a few-mode fiber (FMF), an MSC is constituted to convert the Gaussian-like fundamental mode to HOMs 
[42]. Photonic lanterns are integrated optical components with intriguing ability of generating specific HOMs in different ports. This method of generating HOMs is capable of mode converting with low crosstalk, providing the potential value in SDM system [33]. Periodic refractive index change is applied in fibers to realize mode conversion from forward propagating fundamental mode to forward propagating HOMs in LPGs [35] or backward propagating HOMs in FBGs [39]. These methods can demonstrate high efficiency of mode conversion, but their limited versatility of static generation of spatial modes greatly constrains the application domain of HOM light sources. An effective and widely adaptable solution to generate spatial modes with dynamic switching capability would potentially expand the practical applications of spatial modes.

Acousto-optic mode converters (AOMCs) employ acoustic waves to create acoustically induced fiber gratings (AIFGs), providing a tunable spatial mode generation with high switching speed [43-50]. The pioneered discovery in 1986 that Kim et al. firstly proposed and demonstrated the mode conversion with a frequency shift by a two-mode fiber-based AOMC [43], which has opened a door to novel researches on acousto-optic interactions (AOI) in fibers and various derived components. Among them, the most extensively concerned are tunable filters [51-64], super lattice modulations [65-69], heterodyne vibration detections [70-73], AOI in photonic crystal fibers (PCFs) [74-77], wavelength tunable lasers [78-82], ultrafast fiber lasers based on AOMCs [83-92], vector modes generations [93-97], optical switchers and modulators [98-100], frequency shifters [101-103] and polarization controls [104], as shown in Figure 1. From the development tree of AOMCs, the practical applications well exploit the intriguing properties of dynamic switching and tuning, frequency shifting and mode conversion in AOMC devices. Previously, AOI-based devices for frequency shifting, FBG sideband modulation and tunable filter are the research hotspots. The frequency up-shifting and down-shifting during the mode conversion between fundamental core mode and HOMs provide the possibility of carrying acoustic vibration information for heterodyne detections. The control of microwave radio frequency (RF) signal enables the resonance tunability for dynamic band-reject and band-pass filtering. Later, fiber laser soon found their demand of AOI-based filter device and active modulation technique. Recently, benefit from the new mechanism of the co-effect of acoustic and optical birefringence on the AOMC in an ellipse-core FMF (E-FMF), dynamic mode switching could be demonstrated successfully via frequency shift keying (FSK) technique. The novel AOMC with dynamic mode switching has attracted immense attentions targeting in dynamic optical field tuning for HOM lasers

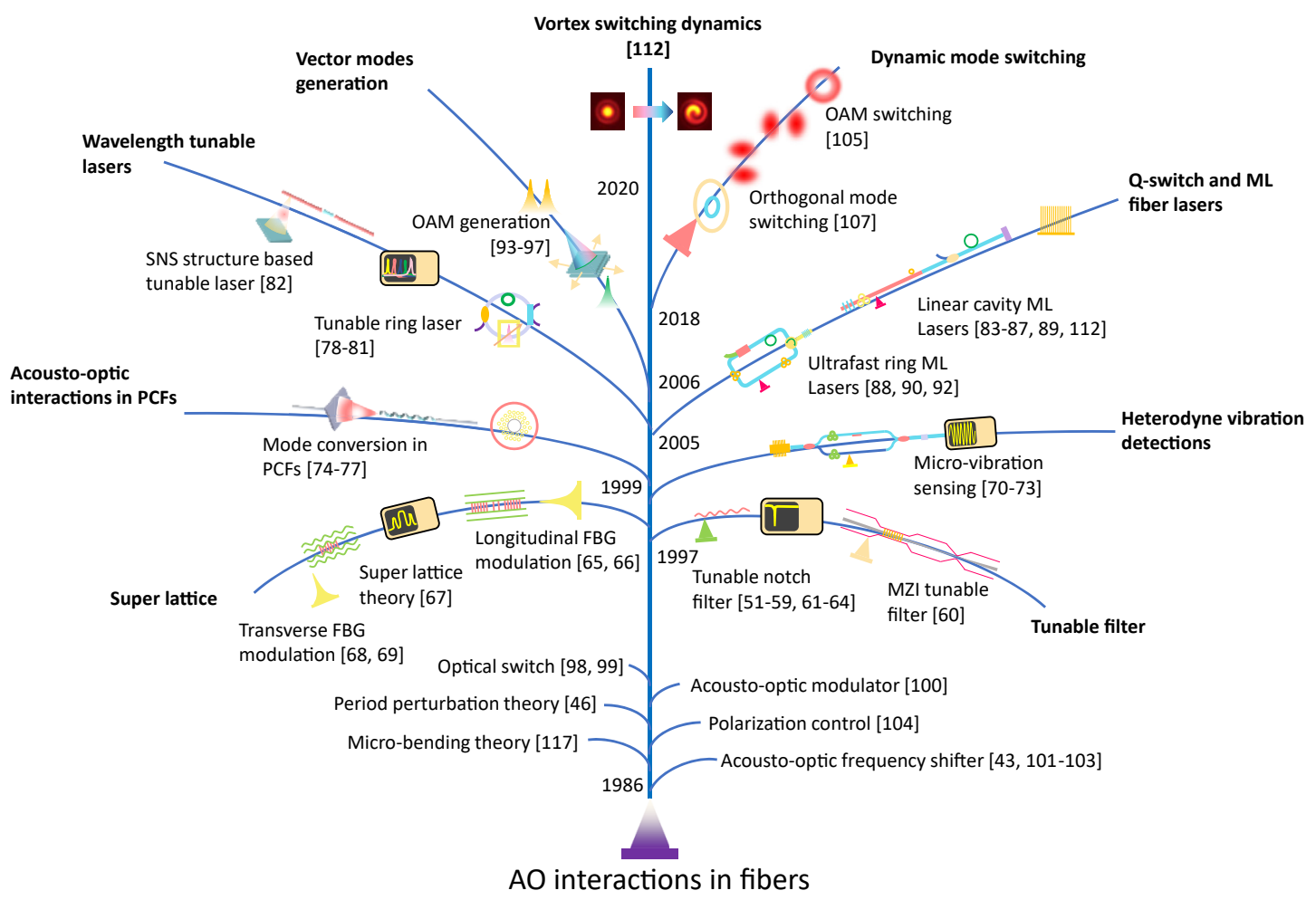

Figure 1: The development tree of researches based on acousto-optic (AO) interactions in fibers. 
[105-111] and spatial mode perturbation for spatial mode locking (ML) mechanism research [112].

In this review, we summarize the new mechanisms of dynamic mode switching based on AOMCs and the applications incorporated with HOM fiber lasers. Firstly, the fundamentals of classical AOMCs and novel dual resonant AOMCs are introduced in Section 2, including the basic mechanism of the AOMC, physical principle of mode switching, and new devices derived from AOMCs. Secondly, dynamic mode switching fiber lasers with continuous-wave (CW) output are described in Section 3. Then, ultrafast fiber lasers incorporating AOMC-based dynamic mode manipulation are specifically commented in Section 4. Mode switching ultrafast fiber lasers possessing complex optical field structures are demonstrated in both spatial and time domains. Furthermore, vortex mode switching dynamics in ML process is introduced and discussed in detail. Finally, the outlook and future challenges of AOMC-based dynamic mode manipulation are discussed in Section 5.

\section{Fundamentals of acousto-optic interactions in fibers}

\subsection{Fundamentals of classical AOMCs}

The advanced discovery reported by Brillouin in 1922 that acoustic waves and light waves can interact has led to appreciable frontiers in acousto-optics. In an optical fiber, the acoustic wave can travel along the unjacketed fiber and the AOI is mainly induced by the light diffraction from a moving refractive index periodic modulation.

From a quantum mechanical view, the photonphonon scattering is deemed as the energy and momentum conservation [50]:

$$
\omega^{\prime}=\omega_{0}+\omega_{a} ; k^{\prime}=k_{0}+k_{a} .
$$

$\left(\omega^{\prime}, k^{\prime}\right),\left(\omega_{0}, k_{0}\right)$ and $\left(\omega_{a}, k_{a}\right)$ are the frequency and the wavenumber of diffracted light, incident light and acoustic wave, respectively, as shown in Figure 2B. In fiber acoustooptics, the practical applications based on acoustically induced refractive index modulation depend on different kinds of acoustic vibrations including longitudinal mode [65-67, 84], torsion mode [113-117] and flexural mode [43, 59, 71, 107-109, 112], as depicted in Table 1.

Longitudinal modes excite acoustic pulses to induce the interlace of medium density, which contributes to the superlattice in FBGs for sideband modulation. The torsion mode of acoustic wave is enabled by exploiting two piezoceramic transducers (PZTs) with inverse vibration directions. Thus, the horn experiences a twisted force with the interlace of clockwise and anticlockwise rotations. The torsion mode of acoustic perturbation exhibits high polarization dependence of mode conversion so that it is widely used in polarization mode generation and filtering. Among these, the flexural mode excites highest coupling efficiency and is capable of mode conversion between copropagating light modes in optical fibers. Especially employing acoustic modulations in FMFs, the AOIs deliver profound possibilities of generating HOMs and orbital angular momentum (OAM) modes with a high degree of freedom in fast mode switching capability, as shown in Figure 2A. With the control of microwave RF signal (both frequency and amplitude), the acoustic vibration can be modulated with different tuning degrees on AOIs. Therefore, arbitrary mode conversions can be demonstrated via controlling the applied RF modulation, as shown in Figure $2 \mathrm{C}$ and $\mathrm{D}$. Previous researches focus on the wavelength tunability of optical filter induced by acousto-optic (AO) effect [53, 57, 59, 62-64, 118, 119]. In recent years, numerous progresses of HOM conversion enabled by the AOMC have been established by a rich variety of theoretical and experimental results [93-97, 105-112, 114, 120].

It is a common belief that the microbending theory is the basic explanation of mode conversion in an AOMC [121]. The flexural acoustic wave (FAW) is utilized to explain the asymmetric vibration induced microbending on fiber medium. The FAW is produced by electrically modulated PZT materials and creates the mode conversion from core fundamental $L P_{0, n}$ mode to high-order $L P_{1, n^{\prime}}$ modes. The lowest FAW mode $F_{11}$ mode propagating on the unjacketed silica fibers induces the periodic density change asymmetric with respect to the direction of acoustic vibration, which can be interpreted by the acoustically induced displacement vector (AIDV) of $\tilde{u}$. The AIDV-based microbending on the fiber medium leads to the permittivity modification $\delta \widehat{\varepsilon}$ composed of the geometric perturbation of $\delta \varepsilon_{g}$ and the photon-elastic effect of $\delta \varepsilon_{p}[50,112]$ :

$$
\begin{aligned}
\delta \widehat{\varepsilon}(x, y, z, t) & =\delta \varepsilon_{g}(x, y, z, t)+\delta \varepsilon_{p}(z, t) . \\
u & =u_{0} \cos \left(k z-f_{a} t\right) \cdot e^{i \frac{2 \pi}{\Lambda}} .
\end{aligned}
$$

$u$ stands for the value of the AIDV. Since the shape of fiber end facet is a circle, the vibration can be assumed to be along $x$ axis. Thus, $\tilde{u}$ is simplified to be $u$ along axis $x . u_{0}$ and $f_{\mathrm{a}}$ are the amplitude and the frequency of the AIDV. $K$ and $\Lambda$ represent the wave vector and the period of AIFG. $\Lambda$ depends on the applied RF signal and is expressed by:

$$
\Lambda=\sqrt{\frac{\pi C_{a} R_{c l}}{f_{a}}} .
$$




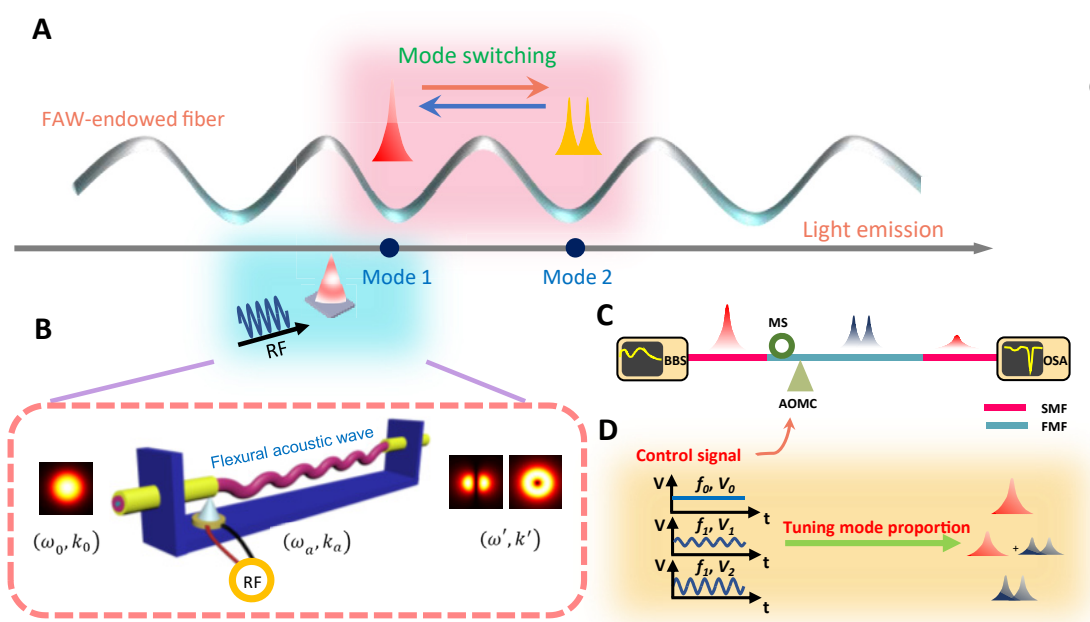

G

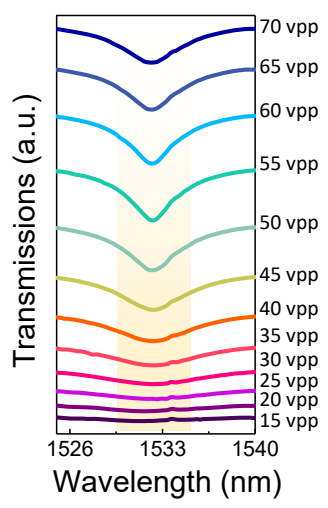

H
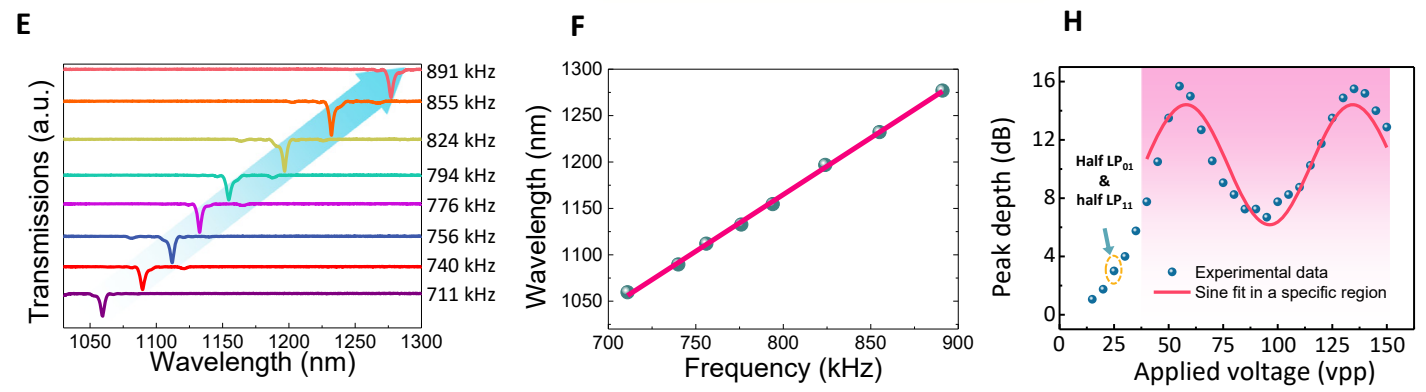

Figure 2: The schematic diagram and characteristics of a classical acousto-optic mode converter (AOMC).

(A) The diagram of mode switching via acousto-optic effect. (B) The experimental configuration of an AOMC device (Reproduced with permission from Lu et al. [105]). (C) The experimental setup for characterizing the dynamic tunability of the AOMC. BBS: broadband optical source. OSA: optical spectrum analyzer. (D) The schematic of mode controlling by radio frequency (RF) signal modulation. (E) The transmission spectra at different applied RF signal frequencies. (F) The variation of resonant center wavelength with the increase of applied RF signal frequency. (G) The transmission spectra at different applied RF signal voltages. $(H)$ The variation of resident fundamental mode power with the increase of applied RF signal amplitude.

$C_{a}=5760 \mathrm{~m} / \mathrm{s}$ represents the velocity of acoustic wave propagating on the fiber medium. $R_{c l}$ stands for the radius of the fiber cladding. Obviously, the dynamic AIFG structure is capable of tunability under the control of applied RF signal. Then the AIDV induced geometric deformation and the photon-elastic effect $\delta \varepsilon_{p}$ of permittivity modifications can be obtained by employing the standard framework of microbending modal:

$$
\begin{aligned}
& \delta \varepsilon_{g}=n_{c o} \cdot K^{2} \cdot u \cdot x \\
& \delta \epsilon_{p}=-\epsilon_{c o}^{2} p \widehat{S}=\epsilon_{c o}^{2} p K u_{0}\left(\begin{array}{ccc}
0 & 0 & 1 \\
0 & 0 & 0 \\
1 & 0 & 0
\end{array}\right) \sin \left(K_{Z}-f_{a} t\right) .
\end{aligned}
$$

$n_{c o}$ is the refractive index of the fiber core. $\varepsilon_{c o}$ is the permittivity of fiber core material. $p$ and $\widehat{S}$ represent the photoelastic coefficient and the strain tensor, respectively.

To obtain the mode conversion, the phase matching condition should be satisfied and the corresponding mode conversion efficiency can be expressed incorporating electric field distributions of the identified spatial modes of $E_{0, n}(x, y)$ and $E_{1, n^{\prime}}(x, y)[95]$ :

$$
\begin{gathered}
L_{B}=\lambda_{c} \cdot\left(n_{0, n}-n_{1, n^{\prime}}\right)^{-1}=\Lambda . \\
\kappa \propto \frac{\pi}{\lambda} \sqrt{\frac{\varepsilon_{0}}{\mu_{0}}} n_{c o} \int E_{0, n}^{*}(x, y) \cdot \delta \widehat{\varepsilon} \cdot E_{1, n^{\prime}}(x, y) d x d y .
\end{gathered}
$$

$L_{B}$ is the beat-length of between fundamental mode and the targeted HOM. $\lambda_{c}$ is the resonant wavelength. $n_{0, n}$ and $n_{1, n^{\prime}}$ are the effective refractive indices of the fundamental mode and HOMs. The AOMC component has the tunability of resonant wavelength shift and intensity modulation. Once the AOMC is fabricated, the only variable to tune the AIFG period is the frequency of the applied RF signal. Figure $2 \mathrm{E}$ and $\mathrm{F}$ shows the changes of the transmission spectra and the resonant center wavelength of a twomode-fiber based AOMC at the varying of RF frequency, 


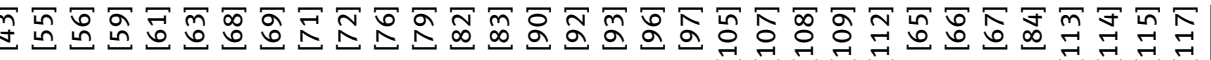

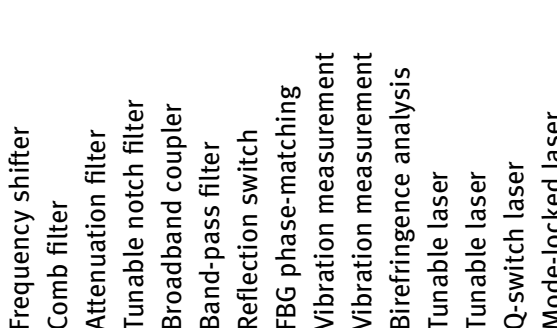

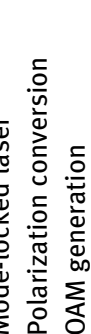

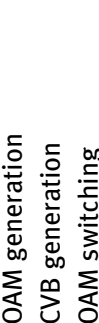

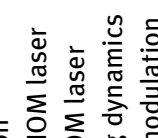

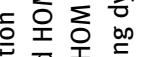

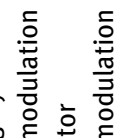

$\frac{\overline{0}}{\frac{\pi}{5}}$

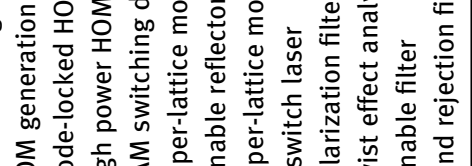

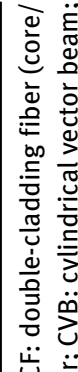
$\frac{2}{0}$

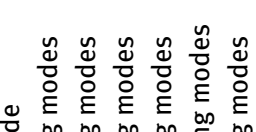

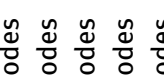

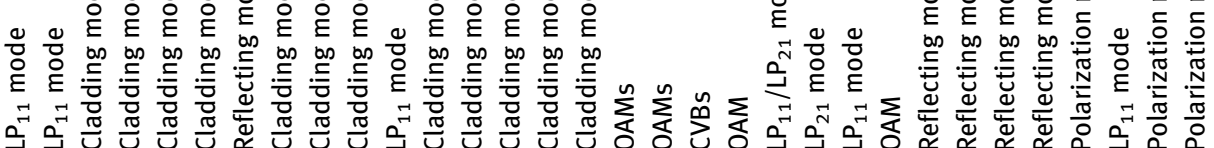

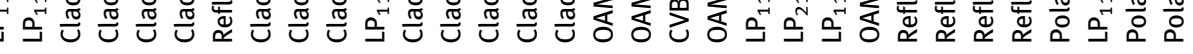

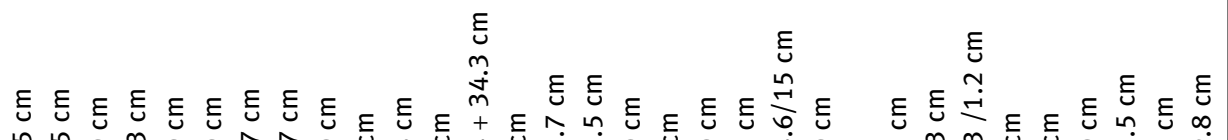

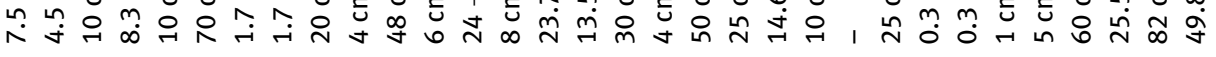


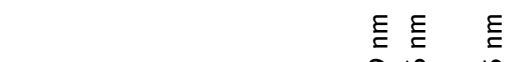

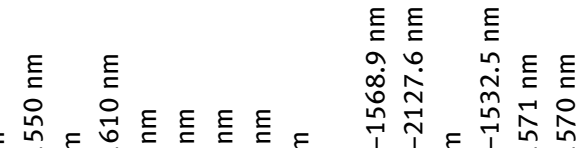

E

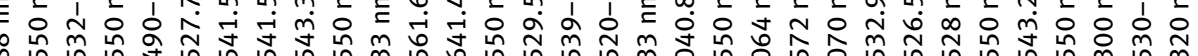

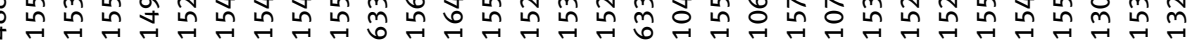

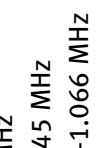

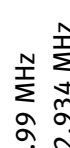

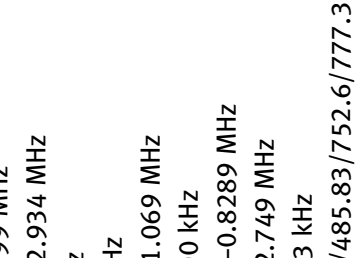

$\varepsilon$

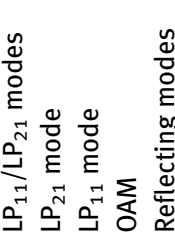

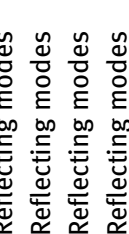

究

$\sum_{i=1}^{i n}$

(1)

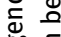

$\stackrel{n}{m}$

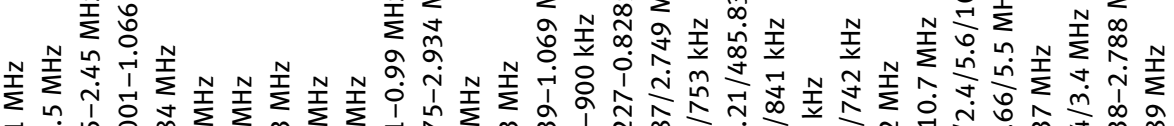
in $i$ 出o 0 n $\rightarrow$ i

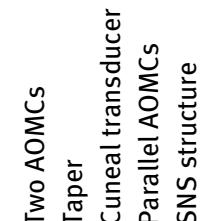

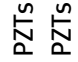
$\frac{n}{\frac{\pi}{N}}$

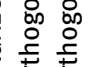

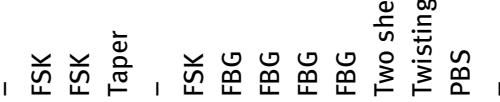

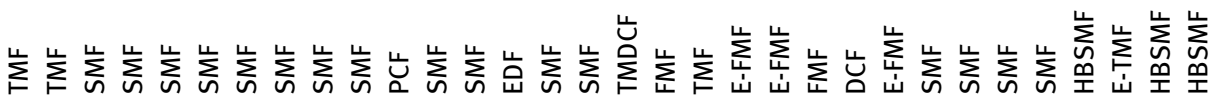

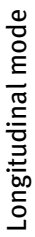

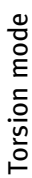
点

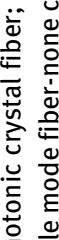
을 $\frac{0}{\mathrm{~m}}$

崖帛 
respectively. From Eq. (8), the mode conversion efficiency can be controlled by changing the RF signal amplitude. It is worth noting that the intensity modulation has different stages at increasing the applied RF signal voltage, as depicted in Figure $2 \mathrm{G}$ and $\mathrm{H}$. When the voltage is at the low region, the $\mathrm{LP}_{01}$ mode power decreases with the increase of the applied voltage, indicating that the energy converted to HOM is increasing. The experimental result shows that when the voltage is over the threshold ( 90 Vpp), the conversion efficiency declines with the increase of the applied voltage due to over-coupling effect.

\subsection{Mechanisms of dual-resonant AOMC for dynamic mode switching}

The classical AOMC has a single resonance of mode conversion between adjacent order modes, for instance, only one resonant wavelength for mode conversion from fundamental $\mathrm{LP}_{01}$ mode to $\mathrm{LP}_{11}$ mode. Recently, a new $\mathrm{AO}$ mode conversion with dual resonance employing a slightly ellipse fiber has been proposed [105]. The irregularity of the fiber induces the HOM degeneration and creates the dual resonance of the AOMC.

A slight ellipticity of the silica fiber in the AOMC induces the co-effect of optical and acoustic birefringence. Firstly, the radius of the fiber cladding is degenerated into a long axis and a short axis. Secondly, the ellipticity causes the acoustic birefringence. Figure 3B shows the ellipsefiber-based AOMC setup and the corresponding fiber end facet model. The acoustic vibration should consider an orthogonal decomposition:

$$
\begin{gathered}
\Lambda=\left[\begin{array}{l}
\Lambda_{l} \\
\Lambda_{s}
\end{array}\right]=\left[\begin{array}{c}
\sqrt{\pi C_{a} R_{l} \cdot\left(f_{a}\right)^{-1}} \\
\sqrt{\pi C_{a} R_{s} \cdot\left(f_{a}\right)^{-1}}
\end{array}\right] . \\
\varepsilon_{0}(x, y) \rightarrow \varepsilon_{0}(x-y) \varepsilon_{0}\left(x-u_{x}, y-u_{y}\right) . \\
u_{x}=u_{0} \cos \left(K_{Z}-f_{a} t\right) \cdot e^{i \frac{2 \pi}{\Lambda_{l}}} ; \\
u_{y}=u_{0} \cos \left(K_{Z}-f_{a} t\right) \cdot e^{i \frac{2 \pi}{\Lambda_{l}}} . \\
\delta \varepsilon_{g}=\left[\begin{array}{l}
\delta \varepsilon_{g}^{x} \\
\delta \varepsilon_{g}^{y}
\end{array}\right]=\left[\begin{array}{l}
n_{c o} K^{2} u_{x} \cdot x \\
n_{c o} K^{2} u_{y} \cdot y
\end{array}\right] .
\end{gathered}
$$

Finally, the ellipticity of the fiber core enables a degeneration of HOMs. Thus, the mode conversion between $\mathrm{LP}_{01}$ mode and $\mathrm{LP}_{11}$ mode has two components and the efficiency can be modified into:

$$
\begin{aligned}
& \kappa(z)=\left[\begin{array}{l}
\kappa_{a}(z) \\
\kappa_{b}(z)
\end{array}\right]
\end{aligned}
$$

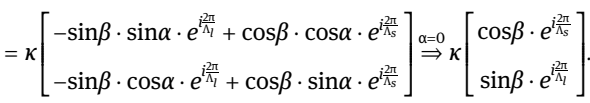

The dual-resonant transmission spectra combined of both $\mathrm{LP}_{11 \mathrm{a}}$ mode and $\mathrm{LP}_{11 \mathrm{~b}}$ mode patterns at different resonant wavelengths in Figure $3 \mathrm{C}$ well confirms a simultaneous orthogonal HOM generation. By setting the frequencies appropriately, the two mode conversions can be coincident at a same resonant wavelength. Furthermore, the dynamic mode switching can be demonstrated by employing the electrically alternated applied frequency via the FSK technique, as depicted in Figure 3A. The beat-lengths between fundamental mode and HOMs are calculated by a finite element method (Comsol Multiphysics). From Figure 3D, the ellipticity $[E=\arctan (x / y)]$ of the fiber determines the strength of the birefringence and further controls the wavelength separation $(\Delta \lambda)$. In Figure 3E, the wavelength separation declines as the decrease of the ellipticity. It well indicates a great possibility of the dualresonant spectrum shaping or the wideband discrimination of HOM generation.

The mode switching time is an important performance of the mode switchable AOMC. The switching time is mainly deemed as the propagating time of the acoustic flexural wave during the AO coupling region [95]:

$$
\tau=L /\left(\pi \cdot R_{c l} \cdot C_{a} \cdot f_{a}\right)^{\frac{1}{2}} .
$$

$L$ is the length of the $\mathrm{AO}$ coupling region. Figure $3 \mathrm{~F}$ shows the experimental configuration of AOMC switching time measuring. The experimental results depicted in Figure $3 \mathrm{G}$ indicate the switching speed of two orthogonal HOMs reaches $4.3 \mathrm{kHz}$. The speed of mode switching is not fast enough for the application in optical communication (generation $\mathrm{GHz}$ level). However, such a fast tuning property enables possibilities in optical field tuning for broadening the flexibility of laser manufacturing, stimulated emission depletion microscopy (STED) and particle manipulation.

\subsection{Higher-order orthogonal mode switching using cascaded AOMCs}

Generally, the AOMC only conducts the mode conversion between two spatial modes with adjacent azimuthal numbers, on account of the phase-matching condition $[122,123]$. However, higher-order modes have the demand 
A

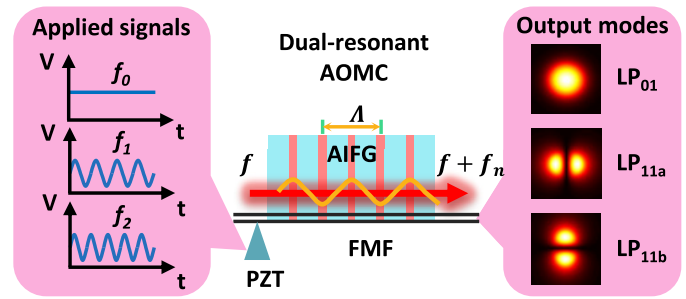

C

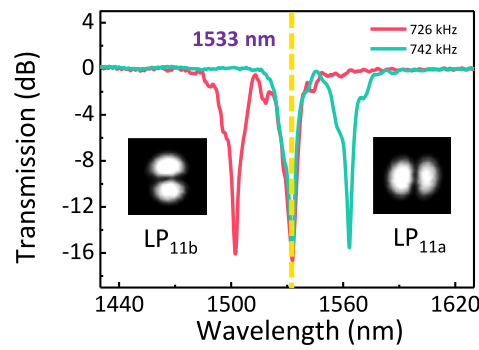

F

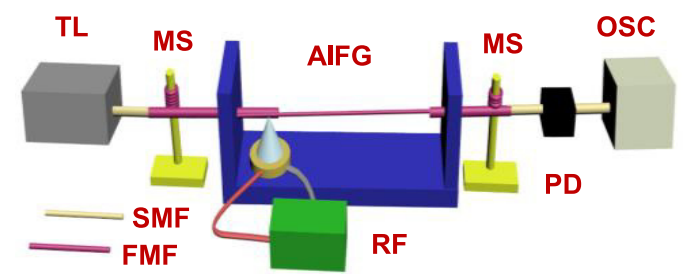

B

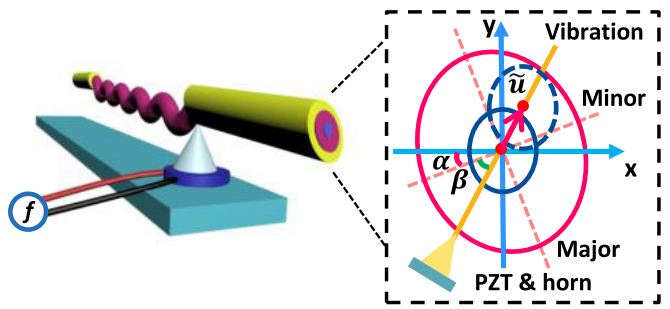

E
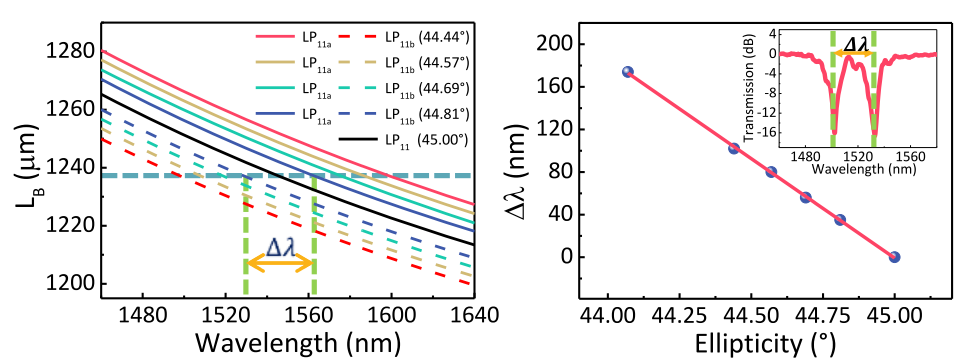

G

Figure 3: The dual resonance of acousto-optic interactions (AOIs) in an ellipse-core FMF (E-FMF) with co-effect of acoustic and optic birefringence.

(A) The schematic diagram of the dual-resonant acousto-optic mode converter (AOMC) and the mode switching mechanism. (B) The model of acoustic and optic birefringence from the view of fiber end face. (C) The dual-resonant transmission spectra at different RF signals incorporating orthogonal $L P_{11}$ mode patterns. (D) The simulated results of orthogonal $\mathrm{LP}_{11}$ mode conversions with different ellipticities of the fiber. (E) The resonant wavelength interval change at the variation of the ellipticity. (A-E are reproduced with permission from Lu et al. [112]). (F) The setup diagram of intensity modulator based on an acoustically induced fiber grating (AIFG). TL: tunable laser; MS: mode stripper; RF: radio frequency; $\mathrm{PD}$ : photoelectric detector; OSC: oscilloscope. (G) The experimental measurement of switching time between different mode $\left(f_{1}: 738.0 \mathrm{kHz} ; \mathrm{f}_{2}: 753.0 \mathrm{kHz}\right.$ ) ( $\mathrm{F}$ and $\mathrm{G}$ are reproduced with permission from Lu et al. [105]).

in diverse applications with the requirements of more intensity centers or higher topological cores. To realize the mode coupling between two modes with nonadjacent azimuthal numbers, cascading two independent AIFGs shows great simplicity and high efficiency, as shown in Figure 4A. The cascaded AOMCs incorporate two AO couplings for constructing two stages of the mode conversions. Besides, both the AOMCs are electrically dominant by the identified RF signals so that the mode coupling from the fundamental mode to several HOMs ( $\left.\mathrm{LP}_{11 \mathrm{a} / \mathrm{b}}, \mathrm{LP}_{21 \mathrm{a} / \mathrm{b}}\right)$ located at a certain resonant wavelength is capable of dynamic control [107].

Due to the co-effect of acoustic and optic birefringence, the simulated wavelength separation between two $\mathrm{LP}_{11}$ $\left(\mathrm{LP}_{21}\right)$ modes is $24(18) \mathrm{nm}$ in Figure $4 \mathrm{~B}$ and $\mathrm{C}$. The two resonances of the orthogonal HOMs under a certain frequency have a wavelength interval of 32(25) $\mathrm{nm}$ for two $\mathrm{LP}_{11}\left(\mathrm{LP}_{21}\right)$ modes. For simplifying the configuration, the cascaded AOMCs can only exploit one frequency $\left(f_{11 \mathrm{a} / \mathrm{b}}\right)$, as shown in Figure 4D. In the configuration, the unjacketed fiber region is divided into two parts with different diameters by hydrofluoric acid (HF) etching with different times. According to Eq. (4), the period of the AIFG is determined by the radius of the fiber when acoustic frequency is fixed. By carefully controlling the diameters of region I and region II, the AIFG periods can satisfy the phase matching conditions of $\mathrm{LP}_{01}-\mathrm{LP}_{11}$ and $\mathrm{LP}_{11}-\mathrm{LP}_{21}$ mode conversion under same applied RF signal, respectively. Therefore, the two AO coupling regions share the same acoustic wave. As shown in Figure 4E, by applying 
A

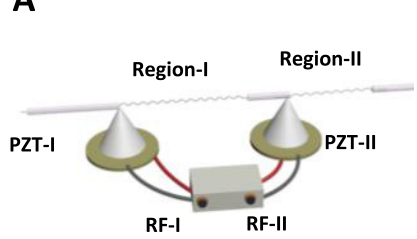

B

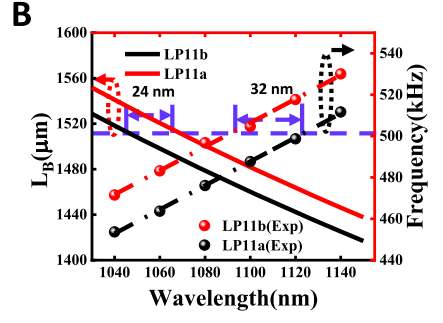

C

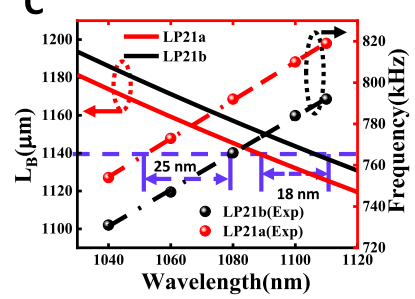

D

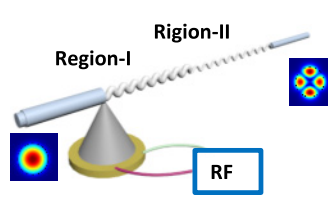

$E$

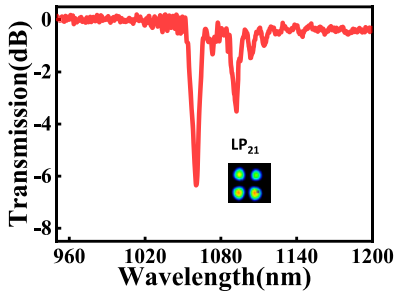

$\mathbf{F}$

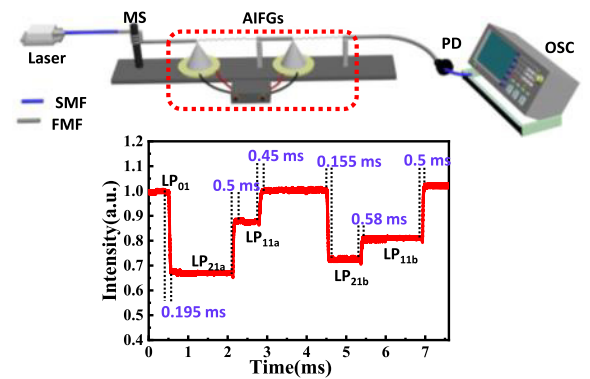

Figure 4: The schematic diagram and characteristics of the cascaded acousto-optic mode converters (AOMCs).

(A) The configuration of the cascaded AOMC device. (B) The beat-lengths and radio frequency (RF) frequency tuning from $L P_{01} m o d e$ to $L P_{11 a / b}$ mode. (C) The beat-lengths and RF frequency tuning from $L P_{11}$ mode to $L P_{21 a / b}$ mode. (D) The configuration of the cascaded AOMC with applying one RF signal. (E) Transmission spectrum of the cascaded AOMC with $\mathrm{LP}_{21}$ mode generation. (F) The schematic setup of the multiple-level intensity modulator (MLIM). (G) Experimental result of the MLIM. The corresponding switching times are measured (Reproduced with permission from Meng et al. [107]).

the frequency $(481.5 \mathrm{kHz})$ on the PZT, the AOMCs can demonstrate the mode conversion from $\mathrm{LP}_{01}$ mode to $\mathrm{LP}_{21}$ mode at $1060 \mathrm{~nm}$.

Figure $4 \mathrm{~F}$ is the schematic diagram of a multiple-level intensity modulator (MLIM) based on the cascaded AOMCs. The mode stripper (MS) ensures a pure fundamental mode into the cascaded AOMCs and the light can be converted to HOMs. Then, the generated HOMs are attenuated in the SMF while the remaining $\mathrm{LP}_{01}$ mode propagates through the SMF and is recorded by the oscilloscope (OSC). With cascading AOMCs, different mode conversions are dynamically controlled. The intensity modulator of multiple level can be fabricated by employing different mode conversion efficiencies, as shown in Figure 4G. The experimental switching time from $\mathrm{LP}_{01}$ to $\mathrm{LP}_{21 \mathrm{a}}\left(\mathrm{LP}_{21 \mathrm{~b}}\right)$ mode is $0.195(0.155) \mathrm{ms}$.

Higher-order modes can be obtained by employing cascaded AOMCs with dynamic control of separate RF signals. The method of cascaded AOMCs benefits from the simple configuration. However, it requires different RF signal generators which increase the cost and complexity of the control system. The method that exploits single RF signal exhibits a compact size for easy integration. But this device lacks the robustness due to the requirement of HF corrosion for fiber diameter control. To date, cascading AOMCs is shown to be the most effective all-fiber method for generating higher-order modes with dynamic flexibility. But the challenge remains how to improve the switching speed with high conversion efficiency.

\subsection{CVM and OAM generations via cascaded AOMCs with orthogonal vibration}

Vector light beams possess doughnut-like light field distributions and anisotropic polarization profiles which have found diverse applications in both scientific researches and industrial designs. Traditional all-fiber method of generating vector modes especially OAM modes incorporates linear polarized HOM conversion and the subsequent light field tuning via changing polarization state and phase difference in FMFs [29]. Due to the inherent birefringence of silica fibers, the light field tuning requires accurate manual adjustment of the polarization controller (PC) which increases the difficulty of the demonstration. Fortunately, cascaded AOMCs are capable of active modulation with accurate phase difference profit from the microwave $\mathrm{RF}$ signal control. Figure 5A delivers the configuration of extracavity vector mode manipulation via cascaded AOMCs. The cascaded AOMCs consist of two PZTs with orthogonal vibration directions and a horn-like transducer. Here, the $\mathrm{x}$-vibration PZT $(f=0.8289 \mathrm{MHz})$ and the $\mathrm{y}$-vibration PZT $(f=0.8227 \mathrm{MHz})$ demonstrate the mode conversions from the linear polarized $H E_{11}^{x}$ mode to $T M_{01}$ mode and $T E_{01}$ mode, 
respectively. The corresponding mode patterns of radially polarized $T M_{01}$ mode and azimuth polarized $T E_{01}$ mode without and with a polarizer are depicted in Figure $5 \mathrm{~B}$ and $\mathrm{C}$, respectively. In this experiment, the utilized FMF possesses a circular morphological structure and relatively large corecladding refractive index difference so that the eigen vector modes $\left(T M_{01}, T E_{01}\right.$, and $H E_{21}^{\text {odd/even }}$, that is, the CVMs) can be degenerated from $\mathrm{LP}_{11}$ mode. By applying different RF signals on the PZT, the certain cylindrical vector beam (CVB) can be generated.

Moreover, when the applied RF signals employ the same frequency of $0.8270 \mathrm{MHz}$, the two PZTs enable the $H E_{21}^{\text {even }}$ mode and $H E_{21}^{\text {odd }}$ mode due to the orthogonal vibration. Dexterously, setting a phase difference of $\pm \pi / 2$ between $\mathrm{RF}_{1}$ (applied on the $x$-vibration PZT) and $\mathrm{RF}_{2}$ (applied on the $y$-vibration PZT) creates a phase shift of $\pm \pi / 2$ on the generated $H E_{21}^{\text {even }}$ mode and $H E_{21}^{\text {odd }}$ mode. Thus, the corresponding \pm 1 -order OAM modes are generated. The \pm 1 -order optical vortex mode patterns and the corresponding off-axial and coaxial interference patterns are shown in Figure 5D and E, respectively.

The orthogonal vibration cascaded AOMC components provide a new pathway for inducing phase difference and lead to the capability of generating the CVBs and the OAMs with different topological cores. This configuration possesses more compact size and more simple generation of vector modes. However, the cascaded AOMCs are unable of avoiding using several RF signals which increases the complexity of the control system. Besides, this method depends on the eigen modes conversion so that it is restricted to the generation bandwidth. Although the dynamic switching of different vector modes is not reported in the demonstration, incorporating the FSK technique can simply realize the dynamic altering between CVBs and OAMs.

\subsection{Low-frequency shifter based on AOMC for microvibration heterodyne detection}

Heterodyne detection, as an optical coherent detection method, has provided a new strategy in high-order harmonic light generating [124], imaging based on nanoclusters and nanocrystals [125], spectroscopy scanning tunneling [126], and gravitational waves detection [127]. An optical heterodyne configuration employs photo-mixing

A
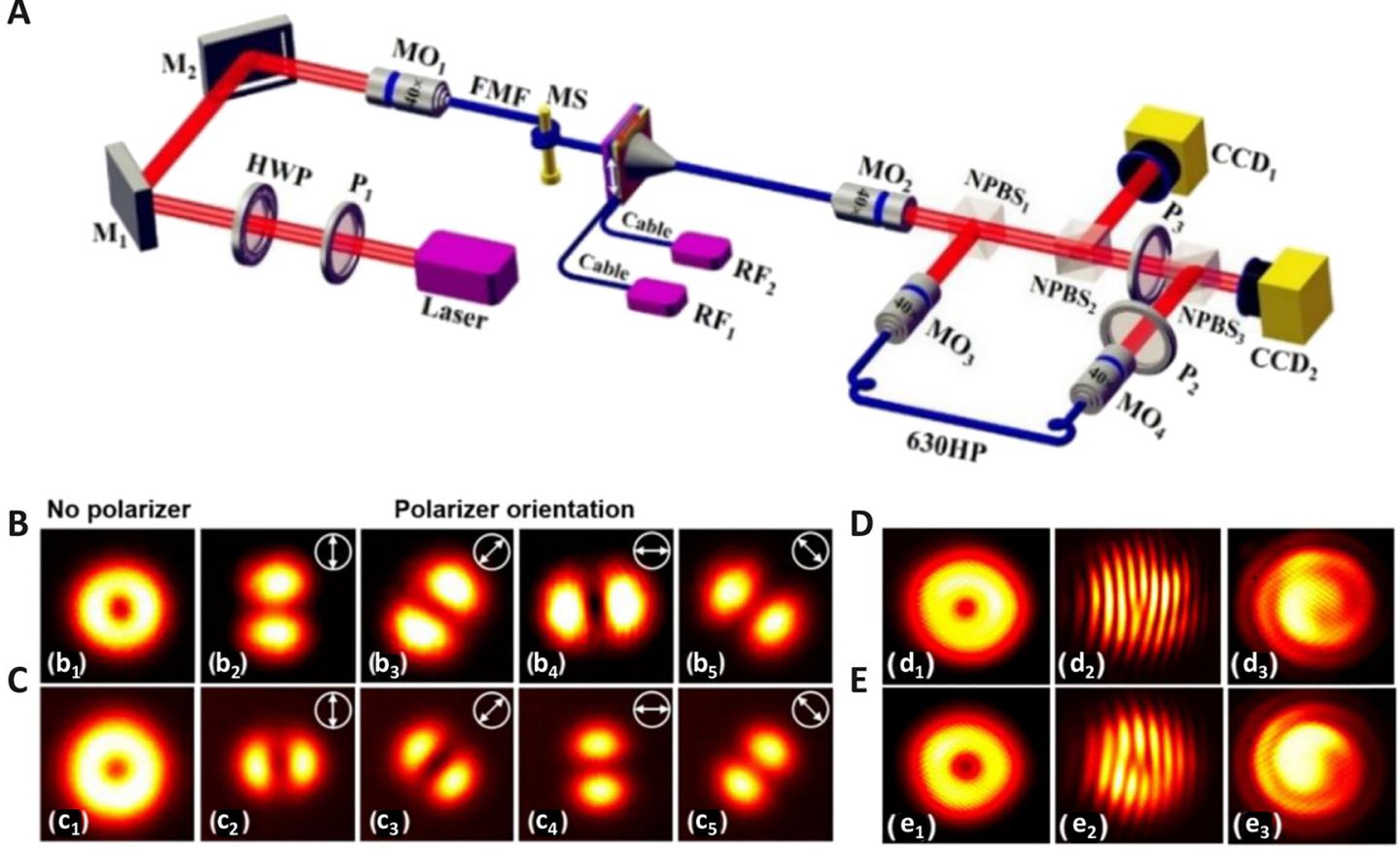

Figure 5: The experimental setup and results of cylindrical vector beams (CVBs) and orbital angular momentums (OAMs) generation based on orthogonal vibration cascaded acoustically induced fiber gratings (AIFGs).

(A) The schematic diagram of the cascaded acousto-optic mode converters (AOMCs) with orthogonal vibration. P: polarizer; HWP: half waveplate; M: mirror; MO: micro-objective; NPBS: non-polarization beam splitter; $630 \mathrm{HP}$ : an SMF at the wavelength of $600-770 \mathrm{~nm}$.

(B) The mode patterns without $\left(b_{1}\right)$ and with $\left(b_{2}-b_{5}\right)$ a polarizer of $T M_{01}$ mode. (C) The mode patterns without $\left(c_{1}\right)$ and with $\left(c_{2}-c_{5}\right)$ a polarizer of $\mathrm{TE}_{01}$ mode. The +1-order OAM (D) and -1-order OAM (E) mode patterns with off-axial and co-axial interferences (Reproduced with permission from Zhang et al. [96]). 
technique, resulting in a down-conversion of probe signal frequency from a high value to a lower, easily measurable one [128]. Thus, the optical frequency shifter creates a highquality frequency shift on the local oscillator signal. However, it is generally challenging for fabricating a single acousto-optic modulator (AOM) through the frequency shift of several hundred kHz. The traditional bulk AOMs like Bragg cell [129] and integrated structure [130], demand precise alignment and high diffraction efficiency. Besides, the performance of these methods for heterodyne detection is restricted by the signal-to-signal beating interference (SSBI) [131].

All-fiber mode conversion incorporating frequency shift provides a potential solution to eliminate such difficulties effectively without the expense of carrier and sideband suppression ratios [132]. The MSC is used for exciting HOMs and removing the SSBI effect in the FMF without the sacrifice of spectral efficiency [131-133], while the AOMC is to reconvert the HOMs back to fundamental mode with an upshifted frequency amount equal to the acoustic frequency $\left(f_{a}\right)$ [43]. Figure 6A shows the all-fiber heterodyne detection with or without the FBG based on mode conversion frequency shifter (MCFS), marked as scheme (A) and scheme (B), respectively. To ensure the carrier signal a good temporal stability, two arms should have the same optical path [72]. Figure 6B and C shows the transmission spectrum and the RF signal of the MCFS, respectively. From the experimental proof, this all-fiber MCFS possesses high performance of pure frequency shift. Compared with freespace devices, it exhibits higher stability for converting microwave information through mode conversion. From Figure 6D, scheme (B) configuration significantly improves the performance of measurement sensitivity than scheme (A). Moreover, Figure 6E indicates that the vibration information can be detected more efficiently by using allfiber FBG heterodyne system. The vibration amplitude of the PZT firstly increases with the increase of the resonant frequencies and then decreases in general as the driving frequency increases. From Figure $6 \mathrm{~F}$ and the inset of Figure $6 \mathrm{E}$, scheme $(\mathbf{B})$ is excellent to increase the measurement sensitivity of heterodyne microvibration detection.

Compared with other devices, MCFS-based heterodyne detection has the advantage of demonstrating lowfrequency shift of kHz-level only by utilizing one single frequency shifter. Benefit from the common mode cancellation technology, MCFS method provides a pathway for removing the SSBI effect. Besides, MCFS exploits core mode conversion which exhibits high conversion efficiency and leads to low loss. Therefore, the MCFS method is capable of high-sensitivity microvibration detection. In addition, an all-fiber configuration also leads to the compatibility of the fiber integration system. However, the MCFS technique has its restrictions due to the requirement of ultranarrow linewidth laser source. Moreover, the lowfrequency shift of the MCFS limits the available measuring range. In addition, the high-order core mode conversion is more easily affected by the polarization state, which requires polarization adjustment and influences the stability of the system. The straightforward method of utilizing

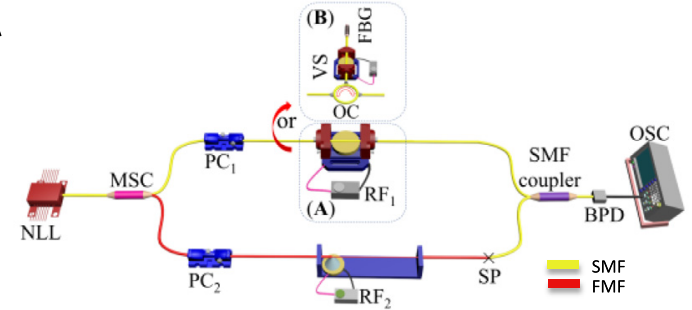

D

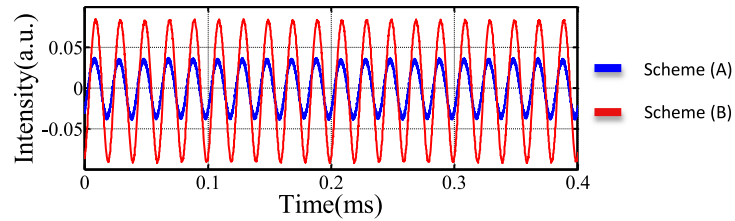

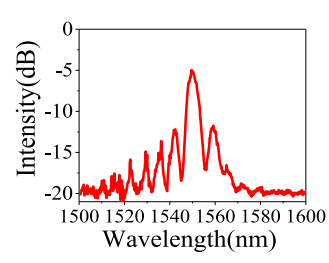

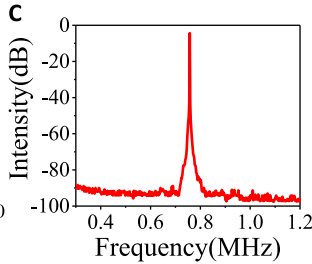

E
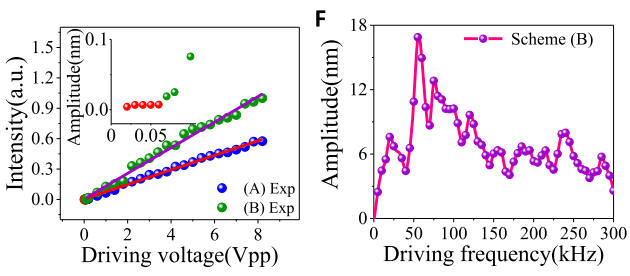

Figure 6: The mode conversion frequency shifter (MCFS) based heterodyne detection system of micro+vibration measurement. (A) Setup diagram of all fiber (A) or all-fiber FBG (B) heterodyne detection based on the MCFS. NLL: narrow linewidth laser; PC: polarization controller; RF: radio frequency; OC: optical circulator; VS: vibration source; SP: splice point between single-mode fiber (SMF) and few-mode fiber (FMF); BPD: balanced photoelectric detector; OSC: oscilloscope. (B) Transmission spectrum of the MCFS. (C) The RF signal of the MCFS. (D) Demodulated vibration waveform. (E) Demodulated amplitude as a function of the driving voltage when $f_{v}=50 \mathrm{kHz}$. (F) Demodulated amplitude as a function of driving frequency $\left(U_{v}=2.5 \mathrm{Vpp}\right)$ (Reproduced with permission from Zhang et al. [132]). 
A

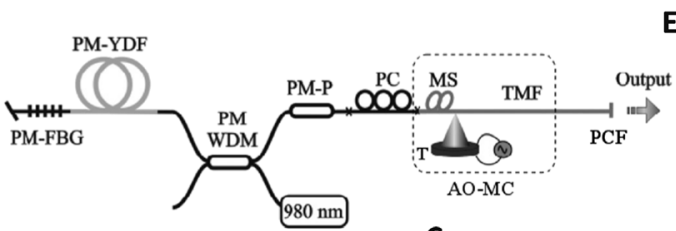

B

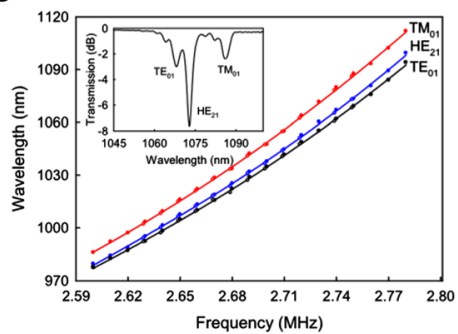

C

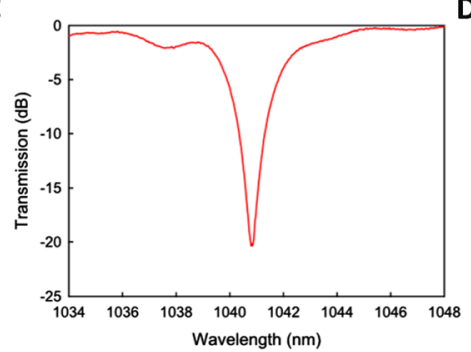

D
$\mathbf{E}$

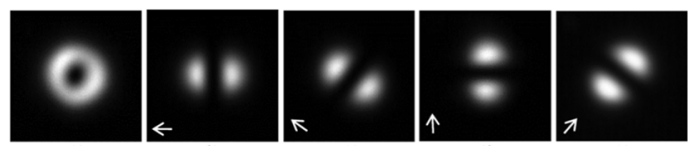

Figure 7: The linear cavity cylindrical vector beam (CVB) laser configuration and the results.

(A) The setup diagram of the linear cavity CVB laser. PM-FBG: polarization maintaining fiber Bragg grating; PM-YDF: polarization maintaining Yb-doped fiber; PM-WDM: polarization maintaining wavelength division multiplexer; PM-P: polarization maintaining polarizer; T: transducer; PCF: perpendicularly cleaved fiber. (B) The simulation of phase matching of cylindrical vector modes (CVMs) with the inset of transmission spectrum of the acousto-optic mode converter (AOMC) excited by unpolarized broadband light source. (C) The transmission spectrum of the AOMC excited by polarization controlled light source. (D) The laser output spectrum. (E) The output TM $\mathrm{TM}_{01}$ mode patterns without and with a polarizer (Reprinted with permission from Carrion-Higueras et al. [97]).

polarization-maintaining fibers (PMFs) is a promising solution in the scheme.

\section{Dynamic mode switching CW fiber lasers}

HOM fiber lasers have attracted tremendous attentions and become a new research hotspot in fiber laser physics in recent years [29, 96, 134-139]. Incorporating fiber laser oscillation and mode conversion outside the laser cavity is the common way to generate HOM laser beams with easy implementation and simple configuration [35, 94, 108]. However, this method is restricted to the low efficiency of HOM laser stimulation. Intracavity mode converters enable the HOM oscillations in the laser cavity, which provides a high-purity HOM laser output $[29,105,109,112,134-136$, 140]. Besides, mode switchable AOMCs enable flexible tunability in spatial degree of freedom to the fiber laser and contribute to wider applications.

\subsection{Cylindrical vector laser enabled by intracavity $A O M C$}

Cylindrical vector light has the advantage of tight focusing due to its intriguing polarization distribution. For example, the radially polarized $\mathrm{TM}_{01}$ mode enables a strong longitudinal electric field component under the focus of a high numerical aperture. Simultaneously its focal spot is far smaller than that of uniformly polarized beam. Such the unique property brings up a broad application prospect in material processing, optical tweezers, excited surface plasma and high-resolution imaging. To create the cylindrical vector light source, an intracavity AOMC is utilized in a linear fiber laser cavity, as shown in Figure 7A. The laser cavity configuration is strictly polarization controlled by employing PMF-based components. The AOMC exhibits triplet resonances of mode conversions from the fundamental mode to the CVMs of $\mathrm{TE}_{01}, \mathrm{HE}_{21}$ and $\mathrm{TM}_{01}$ under an unpolarized broadband light source, as shown in Figure 7B. After polarization control and RF signal setting, the AOMC can convert fundamental mode to $\mathrm{TM}_{01}$ mode at a high efficiency of 99\%, as depicted in Figure 7C. The laser cavity mirror is provided by the FBG reflection and fiber end-face Fresnel reflection. Therefore, the laser oscillates at a narrow bandwidth, as exhibited in Figure 7D. Figure 7E delivers the experimental results of the mode patterns of $\mathrm{TM}_{01}$ mode without and with the polarizer rotation.

The linear cavity of cylindrical vector laser generation includes HOM oscillation which provides a high purity of CVB generation. However, the laser gain is provided by a single mode Yb-doped PMF so that the CVM does not participate in the laser gain. Therefore, the generation efficiency and the purity of CVBs can be further improved by employing HOM laser gain, for instance, using few-mode 
Yb-doped fibers. In addition, the reflection efficiency of the fiber end-face is quite low so that the laser threshold is quite high (100 mW). A pair of FBGs is expected to decline the laser threshold and may decrease the difficulty of laser oscillation. Although the laser output power is moderate (mW-level, lower than general high-power laser output of $\mathrm{kW}$-level), this laser cavity design provides a significant pioneered work for high-power CVB lasers.

\subsection{Dynamic mode switching vortex laser}

It leads to numerous varieties of theoretical and experimental researches of OAM that the pioneered works discover optical vortex carrying topological phase singularity and spiral wave front $[1,4,93,141-143]$. Previous reports on vortex fiber lasers are static generations, that is, the OAM beams with different topological cores are generated separately via manual adjustments $[144,145]$. To extend the flexibility of vortex fiber lasers, dual-resonant AOMC is employed to convert a switchable OAM laser beam [105].

Figure $8 \mathrm{~A}$ shows the diagram of the mode switchable laser cavity that consists of SMF part and FMF part. Highpurity HOMs operate in the laser cavity as follows. Here, the AOMC enables the mode conversions of $\mathrm{LP}_{01}-\mathrm{LP}_{11 a}$ and $\mathrm{LP}_{01}-\mathrm{LP}_{11 \mathrm{~b}}$ by applying different RF signals. The HOMs reflected by the cavity mirror are reconverted to $\mathrm{LP}_{01}$ mode

A

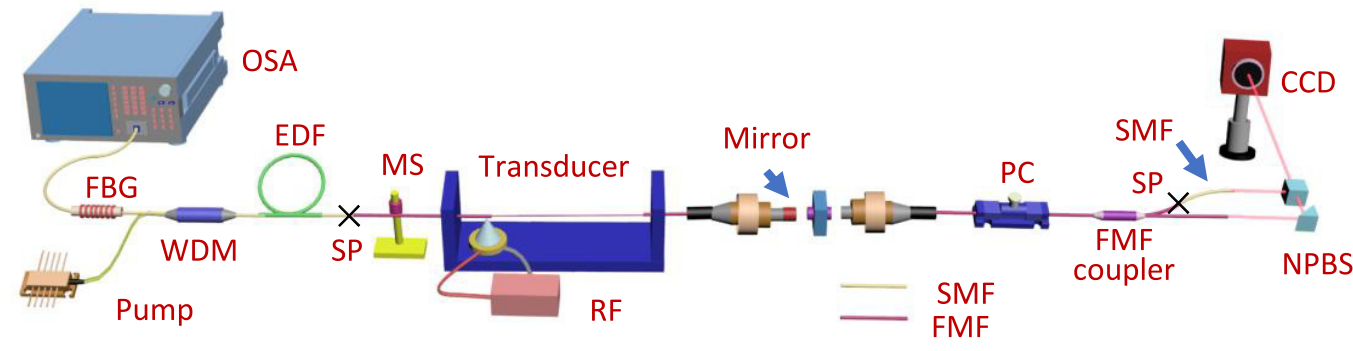

B

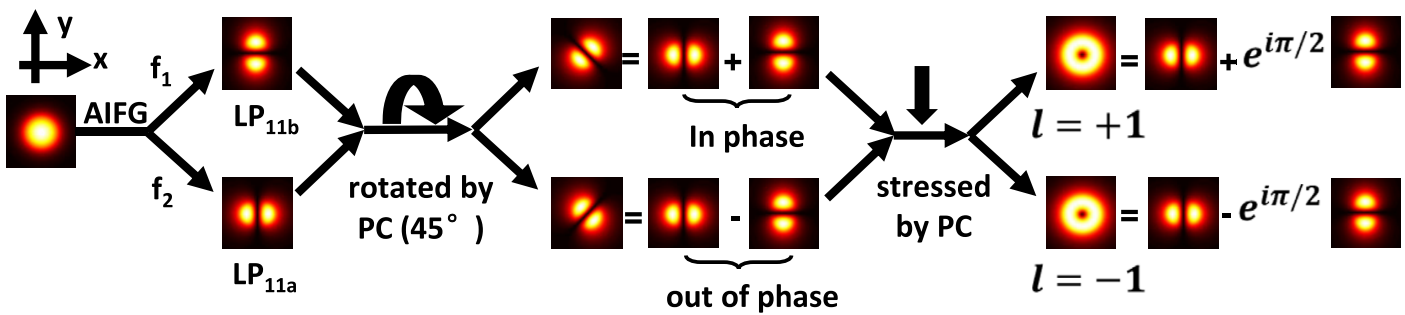

C

$\mathbf{E}$
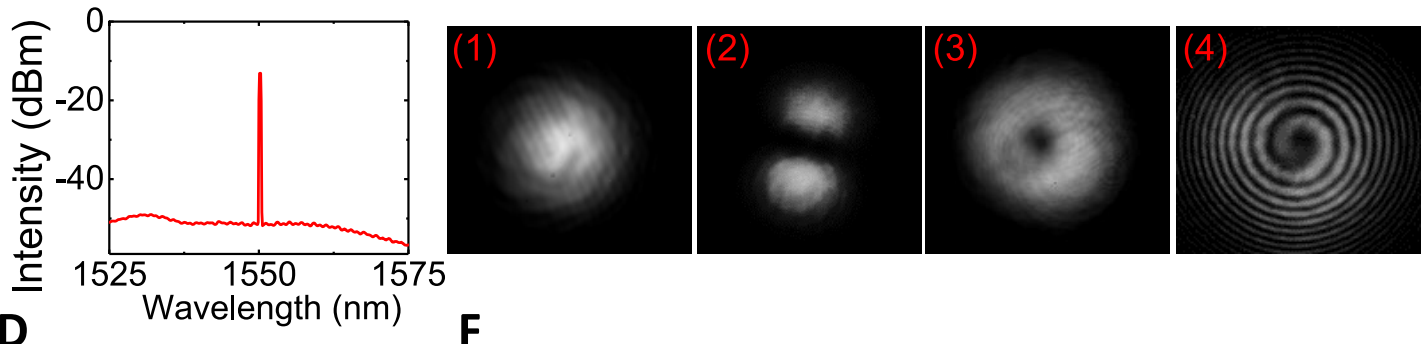

D

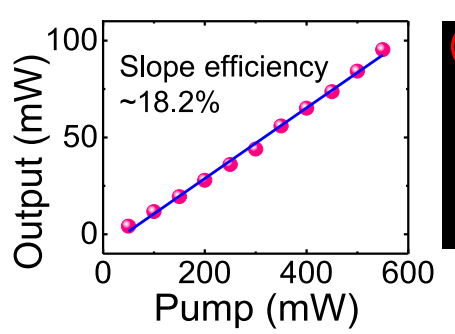

$\mathbf{F}$

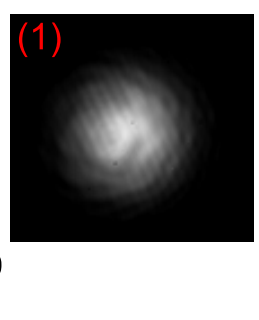

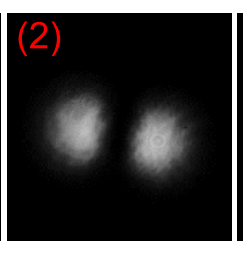
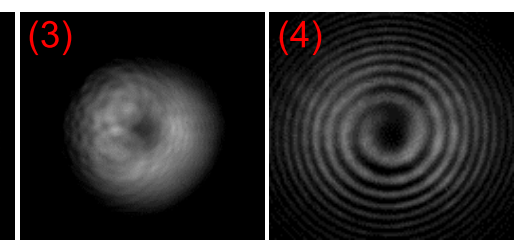

Figure 8: The continuous-wave (CW) fiber laser with switchable orbital angular momentum (OAM) modes output.

(A) The setup diagram of the intracavity CW laser with interference unit. SP: splice point between single-mode fiber (SMF) and few-mode fiber (FMF); PC: polarization controller. (B) The mechanism of switching optical vortex beams (OVBs). (C) The spectrum of CW laser output.

(D) The output power as a function of pump power. The intensity distributions of Gaussian beam (1), $\mathrm{LP}_{11}$ beam (2), OAM beam (3) and interference pattern (4) for $\mathrm{LP}_{11 \mathrm{~b}}$ mode (E) and for $\mathrm{LP}_{11 \mathrm{a}}$ mode (F) (Reprinted with permission from Lu et al. [105]). 
by an AOMC and propagate into the SMF cavity. But the $\mathrm{LP}_{01}$ mode is reconverted to HOMs and dissipated in the splice point before propagating into the SMF cavity. Figure $8 \mathrm{C}$ and $\mathrm{D}$ presents the CW output with the slope efficiency of $\sim 18.2 \%$.

The PC-based optical field tuning technique is employed to obtain the vortex mode switching, as shown in Figure 8B. The generated orthogonal HOMs are firstly rotated at $45^{\circ}$ and then a phase difference of " $\pi / 2$ " (" $i$ ") is obtained by adjusting the stress of the PC appropriately. Finally, the $\mathrm{LP}_{11 \mathrm{a}}$ mode and $\mathrm{LP}_{11 \mathrm{~b}}$ mode are adjusted to be the corresponding -1-order OAM mode and +1-order OAM mode [105, 112]:

$$
\begin{aligned}
& \mathrm{LP}_{11 a(\text { adj })}=\mathrm{TM}_{01}-\mathrm{HE}_{21}^{\text {odd }}-\mathrm{iHE}_{21}^{\text {even }}=\mathrm{OAM}_{-1}, \\
& \mathrm{LP}_{11 b(\text { adj })}=\mathrm{TM}_{01}-\mathrm{HE}_{21}^{\text {odd }}+\mathrm{iTE}_{01}+\mathrm{iHE}_{21}^{\text {even }}=\mathrm{OAM}_{+1} .
\end{aligned}
$$

Once the laser cavity and PC state is adjusted appropriately, the mode switching of OAMs can be demonstrated only by altering the applied RF signal frequency via FSK technique. The corresponding mode patterns of +1 -order OAM and -1-order OAM generation processes are shown in Figure $8 \mathrm{E}$ and $\mathrm{F}$, respectively.

To date, this is the first report of a fiber laser with dynamic switchable OAM modes by using an intracavity AOMC. This configuration exhibits the advantage of OAM switching via electric controlling rather than manual adjusting. Therefore, the dynamic switching of OAM modes has a high speed up to $4 \mathrm{kHz}$. Such a switching OAM light source with different topological cores has the great potentials in diverse scientific researches and industry fields, for example, dynamic optical tweezers and flexible STED imaging. Initially, such an OAM-switchable fiber laser is expected to be a promising light source in mode division multiplexing system for OAM communication. However, the switching speed is restricted to the construction time of the AIFG, which needs to be improved for high-speed optical communication.

\subsection{High-power agile mode fiber laser with AOMC}

The development of high-power fiber lasers has promoted the applications in strong physics research, materials processing, and laser manufacturing [146-149]. Recent years, the HOM high-power lasers have attracted extensive attentions due to their unique intensity, polarization and phase distribution [150,151]. It is a common belief that the mode instability (MI) has become the most important constraint to increase the power of highpower lasers. The foundation of eliminating or reducing the mode instability is to explore the dynamic coupling mechanism of HOMs in high-power lasers. Introducing mode switching into high-power lasers is a significant method to study the dynamic mode coupling mechanism [109].

The experimental configuration of a high-power agile mode fiber laser with intracavity mode switchable AOMC is shown in Figure 9A. Figure 9B shows an obvious result that the output laser beam alters from $\mathrm{LP}_{01}$ mode to $\mathrm{LP}_{11}$ mode at the applied RF signal frequency of $670.0 \mathrm{kHz}$. The corresponding center wavelength shifts from 1070.48 to $1070.07 \mathrm{~nm}$, which matches well with the $\mathrm{LP}_{01}$ and $\mathrm{LP}_{11}$ reflection peaks of the few-mode fiber Bragg grating (FM-FBG).

When raising the pump power, the output power increases with good stability of $\mathrm{LP}_{11}$ mode pattern, as depicted in Figure 9C. The maximum output powers of 6.06 and $5.85 \mathrm{~W}$ at $\mathrm{LP}_{01}$ and $\mathrm{LP}_{11}$ mode lasing are obtained with a slope efficiency of $\sim 48.16$ and $\sim 46.58 \%$, respectively. Benefit from the dynamic tunability of the AOMC, the agile mode high-power laser is capable of generation of highpower laser beam with controllable proportion of HOMs. The output beam profiles under different modulation frequencies are shown in Figure 9D. Figure 9E and F shows the stability of the lasing wavelength and intensity in the mode switchable high-power laser. Although the laser output just reaches several Watts, this demonstration has profound implication for introducing HOM control in highpower fiber lasers. The capability of demonstrating the high-power laser with versatile HOM beam profiles could find potential applications in material processing, light field manipulation.

\subsection{Brillouin fiber laser employing AOMCs}

The pioneered discovery of the nonlinear effect of stimulated Brillouin scattering (SBS) generated in optical fibers contributes to Brillouin lasers with good line-width compression characteristics [153-155], which has found its implications in coherent communication. Brillouin lasers have their unique property of Brillouin frequency shift toward distributed optical fiber sensing, for instance, the Brillouin optical time domain meter (BOTDR) [156, 157]. Moreover, Brillouin lasers exhibit a high signal-to-noise ratio (SNR), showing the promising values in practical applications with low noise requirement $[158,159]$. However, Brillouin lasers have their restrictions of high pumping threshold and low output power. Fortunately, Brillouin Erbium (Er)-doped fiber laser (BEFL) emerges to tackle the bottleneck in the conventional Brillouin lasers [160]. BEFL exploits stimulated radiation of the Er-doped 
A

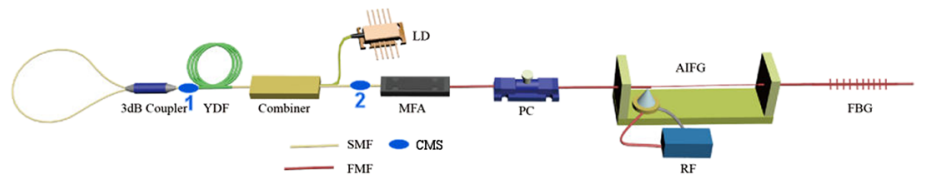

B
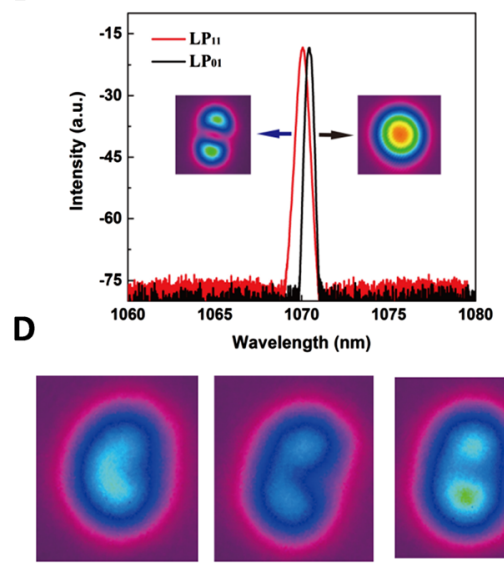

$f=664.3 \mathrm{kHz}$

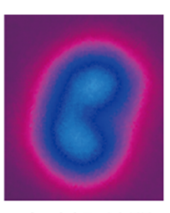

$f=665.1 \mathrm{kHz}$

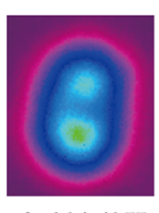

$f=666.4 \mathrm{kHz}$
C

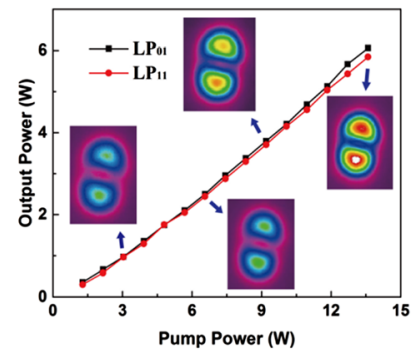

E

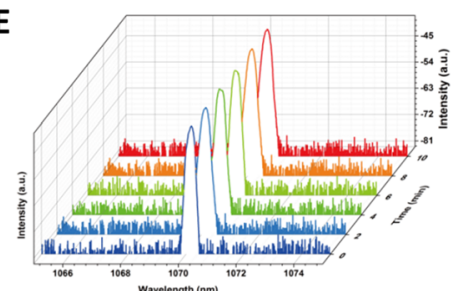

$\mathbf{F}$

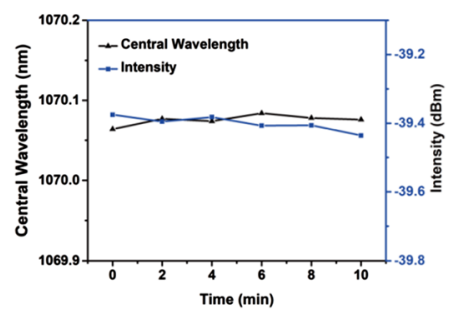

Figure 9: Agile mode switching fiber laser configuration and outputs.

(A) Experimental configuration of the mode-switchable fiber laser. LD: laser diode, YDF: ytterbium-doped fiber, MFA: a home-made mode field adapter via thermally expanded core technique [152]; CMS: cladding mode stripper. (B) The laser spectra of fundamental mode and LP ${ }_{11}$ mode. (C) The output power of $\mathrm{LP}_{01}$ and $L \mathrm{P}_{11}$ mode with different pump powers. (D) The mode profiles at different applied frequencies. (E) The time stability of the agile mode fiber laser. (F) The variation of laser wavelength and intensity over time. (Reproduced with permission from Wu et al. [109]).

fiber for generating high laser energy at $1550 \mathrm{~nm}$ to free the threshold limit of the seed laser.

To date, previous works mainly focus on compressing the line width of the BEFLs through shortening the cavity length and fabricating BEFLs with multiwavelength output. Few works of Brillouin lasers with HOMs output has been reported. Heng et al. have demonstrated the Brillouin lasers with switchable HOMs by using MSCs [161]. However, the mode conversion purity should be further improved because the out-of-cavity mode conversion exhibits low efficiency. Wang et al. employ the photonic lanterns for HOMs generation in Brillouin lasers [162], but the linewidth is restricted to the long cavity length. Recently, a dynamic mode switchable Brillouin fiber laser based on an AIFG provides a new pathway for generating a Brillouin HOM beam with low threshold and narrow linewidth [163].

The dynamic switch of HOM output is enabled by the AOMC incorporating an MSC. The tunable laser source (TLS) serves as Brillouin pump while the $980 \mathrm{~nm}$ pump is utilized to decline the Brillouin threshold, as shown in Figure 10A. From Figure 10B, the spatial mode evolution of light propagating in the laser cavity obviously exhibits the generation mechanism of Brillouin lasing. Firstly, the $\mathrm{LP}_{01}$ mode generated by TLS is amplified by the EDFA and then converted to $\mathrm{LP}_{11}$ mode by the MSC. Meanwhile, the SBS is generated in the FMF by energy accumulation as the Brillouin threshold. The Brillouin laser beam propagates as a counterclockwise direction in the laser cavity so that it is reconverted to $\mathrm{LP}_{01}$ mode when passing through the MSC from the FMF port. Finally, the $\mathrm{LP}_{01}$ mode Brillouin laser beam propagates into the AOMC through the circulator and is converted to $\mathrm{LP}_{11}$ mode with a frequency shift. Figure 10C and $D$ shows the Brillouin laser output under a Brillouin pump (BP) power of $10 \mathrm{~mW}$ and the Brillouin output spectra of both $\mathrm{LP}_{11}$ mode and $\mathrm{LP}_{01}$ mode, respectively. The lasing wavelength of the output spectrum is dynamically controlled by modulating the applied RF signal on the AOMC.

The intracavity AOMC-based Brillouin fiber laser not only has the advantage of emitting ultranarrow bandwidth laser beams, but also possesses the superiority of dynamic switching of the output spatial modes. The HOM Brillouin laser with dynamic tunability is promising in highsensitivity interferometers which can be found in fiber sensing strategy. Furthermore, benefit from the wavelength 

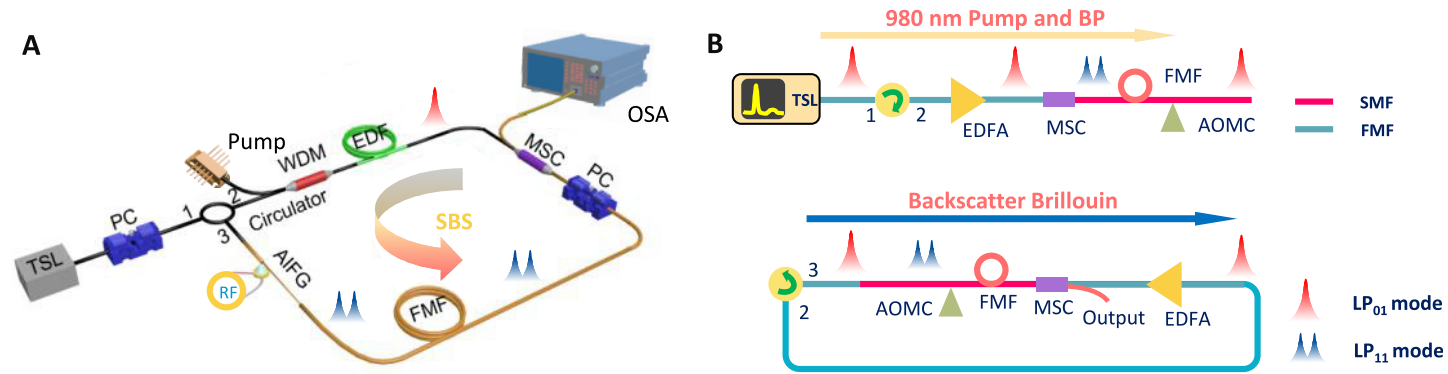

C
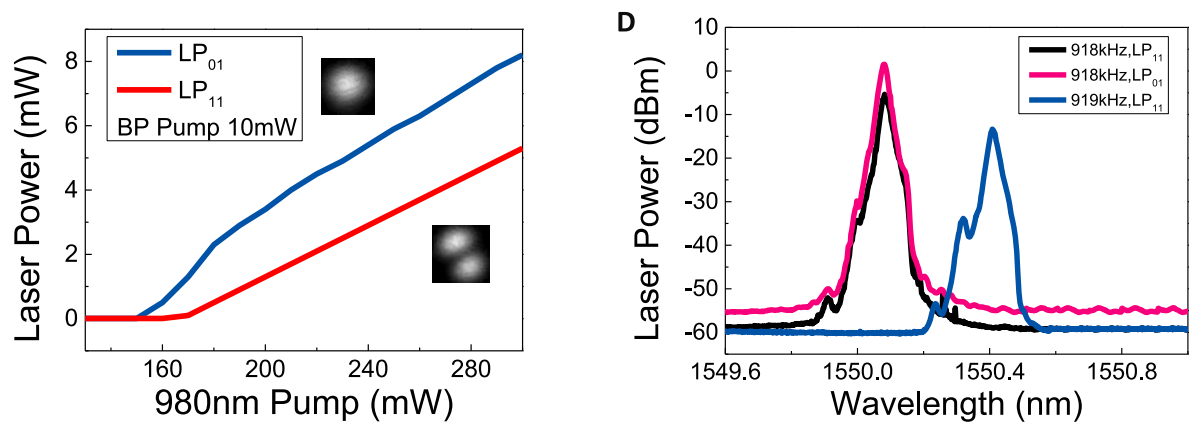

Figure 10: Brillouin fiber laser with dynamic mode switch using the acousto-optic mode converter (AOMC).

(A) The experimental configuration of the dynamic mode-switchable Brillouin laser. (B) The spatial mode evolutions of pump light and Brillouin light in the laser cavity. (C) The Brillouin laser outputs of fundamental mode and $L P_{11}$ mode with the increase of pump power. (D) Brillouin laser spectra of fundamental mode and $\mathrm{LP}_{11}$ mode (Reproduced with permission from Xu et al. [163]).

tuning property of the AOMC, Brillouin laser can oscillate at different resonant wavelengths by exploiting synchronous control of the BP and the applied RF signal frequency. Wavelength tunability can greatly extend the practical applications of the Brillouin laser. A narrow band filter is expected to ensure that $980 \mathrm{~nm}$-pump light excites the Brillouin lasing efficiently. Thus, the Brillouin lasing threshold is reduced and the length of the FMF can be declined for generating a narrower linewidth. Besides, the wavelength tuning property of the AOMC needs to be promoted for continuously adjusting the lasing wavelength of the Brillouin laser.

\section{Ultrafast mode switching fiber lasers}

HOM fiber lasers with CW outputs possess unique symmetry of intensity distribution, polarization property and phase profile [164] and have found their applications in advanced optical communication [165], particle manipulation [166] and nanoscale imaging $[167,168]$. Nevertheless, ultrafast fiber lasers employing ML techniques have their unique properties of ultrahigh peak power, ultrashort pulse duration. Recent years have seen the fast developments and widespread applications of HOM ultrafast fiber lasers [169, 170]. Surprisingly, the HOM oscillation in a mode-locked laser cavity exhibits more interesting phenomena and intriguing properties due to the nonlinear interactions on the ultrafast time scale [171].

\subsection{Ultrafast higher-order modes switching using AOMCs}

To date, AOMC has been well proved to be an efficient method to produce CVBs and vortex beams $[94,96,105]$. Except for the direct generation of vector beams, transverse-mode switching using an AOMC is attracting more attentions. Naturally, mode conversion process of the AOMC can be controlled, and the mode switching between fundamental mode ( $\mathrm{LP}_{01}$ mode) and HOMs contributes to more possibilities of light field manipulation. Currently, AOMC combined with FM-FBGs is the most common way to excite a specific HOM [109]. However, this method is not suitable for generating higher order modes, for instance, $\mathrm{LP}_{21}$ mode. This is because of the relatively low efficiency for the excitation of $\mathrm{LP}_{21}$ mode in FM-FBGs. In addition, 
owing to the narrow-band reflective peaks of the FM-FBG, mode switching of ultrashort pulse cannot be realized.

HOMs switching of ultrashort pulse is demonstrated by using a cascading mode converter incorporating an MSC and an AOMC [108]. From Figure 11A, the mode conversion process has two steps. Firstly, the fundamental mode emitted from the laser source is coupled to linearly polarized $\mathrm{LP}_{11}$ mode by using the MSC, and then $\mathrm{LP}_{11}$ mode is converted to $\mathrm{LP}_{21}$ mode in the AIFG region. In Figure 11B, the mode conversion of $\mathrm{LP}_{21}$ mode is achieved at $1570 \mathrm{~nm}$ when the driven frequency is adjusted to $830 \mathrm{kHz}$. In the experiment, the FMF is etched by $\mathrm{HF}$ ( $40 \%, 30 \mathrm{~min})$ under the room temperature, and the corresponding fiber diameter is reduced to $88 \mu \mathrm{m}$. As shown in Figure 11C, the visibility of the resonant peak reaches $-10 \mathrm{~dB}$, which implies the mode coupling efficiency is $90 \%$.

A real-time mode switching of ultrashort pulses can be obtained by dynamic modulation of the period of the AIFG. The ultrashort pulse is delivered from a home-made ML Erdoped fiber laser, as shown in Figure 11D [172]. The formation of the ML pulse is based on the nonlinear polarization rotation (NPR) effect. Figure $11 \mathrm{E}-\mathrm{G}$ shows the properties of the soliton ML laser. The output laser mode is modulated between $\mathrm{LP}_{11}$ mode and $\mathrm{LP}_{21}$ mode by altering the $\mathrm{RF}$ signal, as shown in Figure 11H. The result shows a mode switching between $\mathrm{LP}_{11}$ mode and $\mathrm{LP}_{21}$ mode. However, HOM conversion efficiency still requires to be improved. Besides, HOM generation employs the MSC to demonstrate the mode conversion from $\mathrm{LP}_{01}$ mode to $\mathrm{LP}_{11}$ mode. Therefore, HOMs can be switched between $\mathrm{LP}_{11}$ mode and $\mathrm{LP}_{21}$ mode.

It is found that extra-cavity AOMCs can also demonstrate the OAM generations from a fundamental mode ultrafast pulsed laser [91, 136]. Figure 12A and B (Figure 12C and $\mathrm{D}$ ) delivers the experimental configuration and the corresponding results of femtosecond (picosecond) pulsed OAM generation, respectively. These configurations exploit the similar strategy for generating OAM pulses by utilizing AOMCs. The experimental proofs well demonstrate the great ability of generating OAMs efficiently. It is worth noting that the optical frequencies of the reference light and interference light should be equal in an OAM detection system via interference method. However, the AO effect induces a frequency shift. Generally, there are two methods for ensuring the frequencies of the reference light and interference light equal. One is to add a same RF signal to the reference light through a phase modulator, as depicted in the configurations of Figure $12 \mathrm{~A}$ and $\mathrm{C}$. The other method is to divide the converted OAM beam into two parts: one is regarded as the interference light; another is regarded as the reference light after splicing to an SMF [105].
A

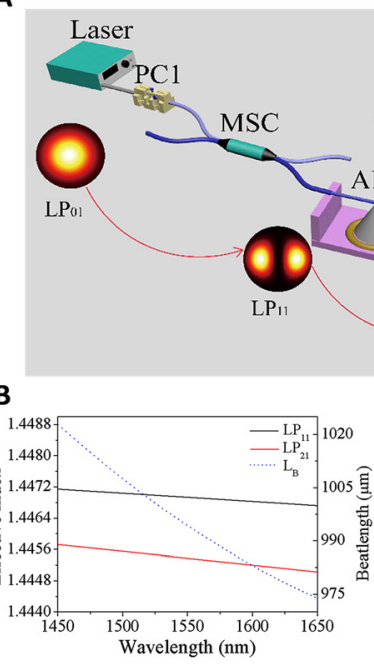

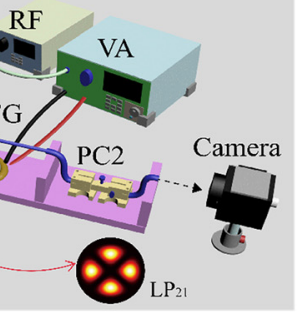

C

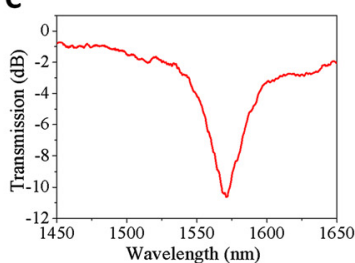

D

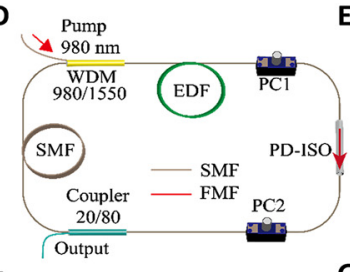

$\mathbf{F}$

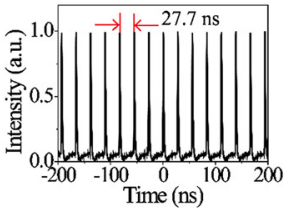

E

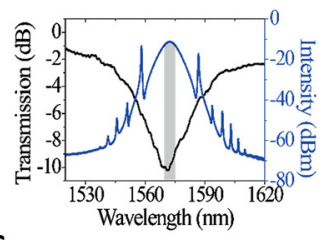

G

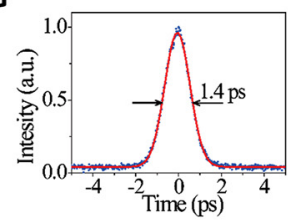

H

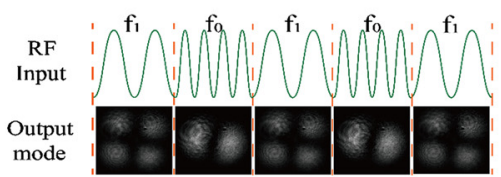

Figure 11: Ultrafast high-order mode (HOM) fiber laser employing cascaded mode selective couplers (MSC) and acousto-optic mode converter (AOMC).

(A) The experimental configuration of the cascading mode converter. VA: voltage amplifier. (B) The simulated mode effective indices and beat-length with $L P_{11}$ and $L P_{21}$ modes. (C) Transmission spectrum of the cascading mode converter for $L P_{21}$ mode generation. (D) The experimental setup of the homemade mode locking $(\mathrm{ML})$ fiber laser. $(\mathrm{E}) \mathrm{ML}$ laser spectrum (blue curve), and the transmission spectrum of the acoustically induced fiber grating (AIFG) (black curve). (F) Pulse train of the ML laser output. (G) Autocorrection measurement of the ML pulse. $(\mathrm{H})$ Real-time switching between $\mathrm{LP}_{11}$ and $\mathrm{LP}_{21}$ modes (Reproduced with permission from Shi et al. [108]). 
A

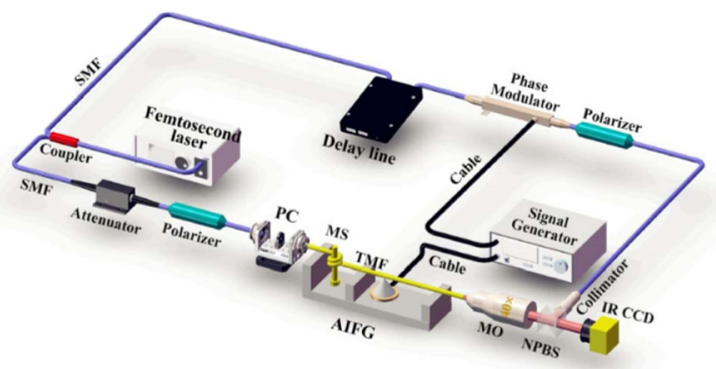

C

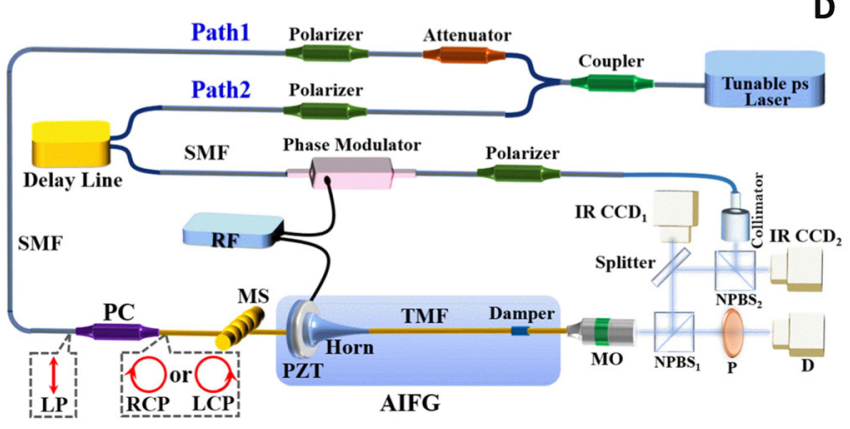

B

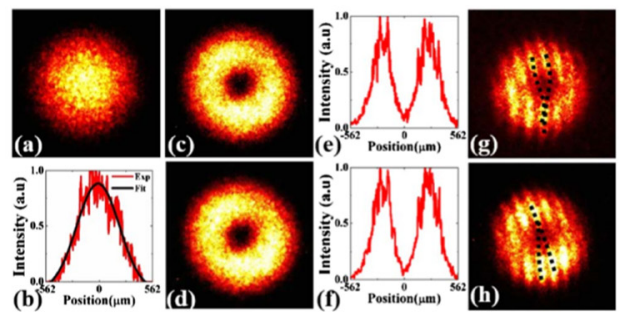

D

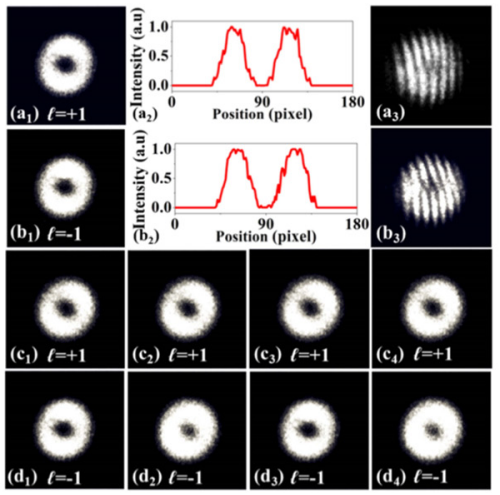

Figure 12: Extra-cavity orbital angular momentum (OAM) generation from ultrafast pulsed laser via acousto-optic mode converters (AOMCs). (A) The experimental configuration of extra-cavity femtosecond OAM generation. MS: mode stripper; TMF: two-mode-fiber; MO: microobjective; NPBS: non-polarization beam splitter; IR-CCD: infrared charge coupled device. (B) The femtosecond fundamental mode pattern (a), distribution (b); +1-order OAM mode pattern (c), distribution (e), interference pattern (g); -1-order OAM mode pattern (d), distribution (f), interference pattern (h). (A and B are reprinted with the permission from Zhang et al. [136]) (C) The experimental configuration of extra-cavity picosecond OAM generation. LP: linear polarization; RCP/LCP: right/left circular polarization; P: polarizer. (D) The picosecond OAM outputs. $\left(a_{1}\right),\left(b_{1}\right)$ Far field modes intensity patterns of the \pm 1 -order OAM modes at wavelength of $1550 \mathrm{~nm} ;\left(a_{2}\right),\left(b_{2}\right)$ horizontal intensity profiles of the \pm 1 -order OAM mode; $\left(a_{3}\right),\left(b_{3}\right)$ fork-like interference patterns when off-axially interfered between the \pm 1 -order OAM modes and linearly polarized Gaussian beam, respectively; $\left(c_{1}\right)-\left(c_{4}\right),\left(d_{1}\right)-\left(d_{4}\right)$ mode intensity patterns of \pm 1 -order OAM modes at wavelength of $1540,1545,1555$, and $1560 \mathrm{~nm}$, respectively. ( $C$ and $D$ are reprinted with the permission from Zhang et al. [91]).

Ultrashort laser pulse with switchable HOMs has great potentials in industrial applications due to its output robustness and simple configuration. However, extra-cavity HOM generation has the restriction of mode conversion efficiency because the HOM does not participate in the laser oscillation. The extra-cavity mode conversion of ML pulses is unable to modulate the nonlinear effects in the laser cavity oscillation. In fact, the AOMC is an effective active modulator for dynamically controlling the laser oscillation especially the ML fiber laser. The mode conversion in CW laser only influence the energy distribution of each mode for laser oscillation. However, the dynamic mode manipulation in a ML fiber laser affects the mode competition on both the spatial domain and time domain in the laser oscillation process. There exist more interesting phenomena of nonlinear effects in the ML laser cavity.

\subsection{Wavelength tunable mode-locked fiber laser via AOMC}

Taking advantage of the spectral tunability, wavelength tunable fiber laser has been proved as a potential seed laser source benefiting numerous applications in optical communication [173], optical sensing [174], materials processing [175], spectroscopy [176] and signal processing [177]. Generally, a tunable spectral filter is required to achieve the lasing wavelength tuning ability such as Fabry-Perot interferometer (FPI) [178, 179], Mach-Zehnder interferometer (MZI) [180], graphene-coated filter [181], chirped FBG [182], W-shape LPG [183] and fiber birefringence filter [184, 185]. Recently, an NPR strategy incorporating intracavity polarizer and intrinsic fiber birefringence has been reported to enable a wide spectral tuning range [186]. These tuning methods are mainly the passive means 
with great depends on laser operation, which are restricted to the tuning range and the response time. Fortunately, AOMC has emerged as an active spectral modulator to contribute the wideband continuous tunability $[78,82$, 187]. Huang et al. have demonstrated tunable fiber lasers employing AIFG-based MZI and incorporating AIFG and a tapered fiber $[79,80,188]$. They also utilize the AIFG filter to generate wavelength tunable vector lasers, which yields extensive attentions in fiber laser researches [92]. Recently, the spectral filtering of a wavelength tunable bandpass filter (TBPF) employing fiber core modes conversion has been proved to exhibit an ultralow optical loss and becomes a new strategy of spectral tuning. Especially, the core mode conversion-based filter incorporating an MSC and an AOMC is capable of simultaneous wide tuning range and fast active response [189].

The configuration of this TBPF is shown in Figure 13A. To generate a highly efficient bandpass filtering property, the resonant wavelengths require fine precise matching. Due to the wideband property of the MSC, the wavelength tunable range of the TBPF can obtain hundreds of nanometers [190-192]. The bandpass property of the TBPF is shown in Figure 13C. AOMCs have been used as filters based on the condition of phase matching [193]. To carry out the wavelength tunable ML fiber laser, the TBPF is employed in the laser cavity providing a tunable filtering mechanism. In addition, the TBPF also plays a role of a splitter in the passive ML fiber laser, as shown in Figure 13C. Figure 13D-F presents the output properties of the ML fiber laser. The TBPF-based ML laser exhibits great wavelength tunability with precise active positioning benefiting from the dynamic control of AOI. This band-pass filter has the advantage of high conversion efficiency and low loss because of employing core mode conversion inside the FMF. Based on the dynamic wavelength tunability of the AOMC, the ML fiber laser is capable of oscillating at different wavelengths. Thus, an intracavity TBPF-based ML fiber laser has more flexible wavelength tuning characteristics than a wavelength tunable ML laser through manual gain adjustment.

\subsection{Vortex mode switching dynamics in an ML fiber laser}

Transient dynamic process in a ML fiber laser delivers the landscapes of the ultrafast pulse evolution. In a ML fiber laser, the ML state is established on the ultrashort transient time scale. The ML fiber laser experiences a series of instabilities before the ultimate ML state [194-201].
A

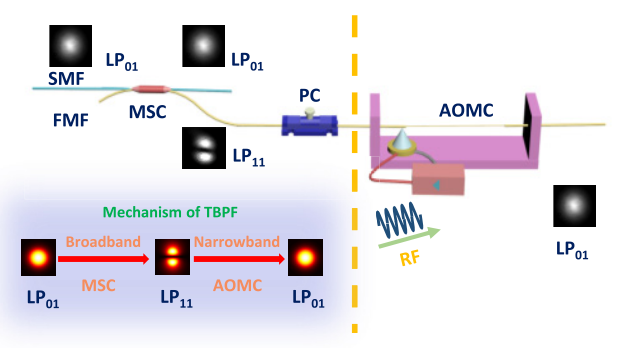

C

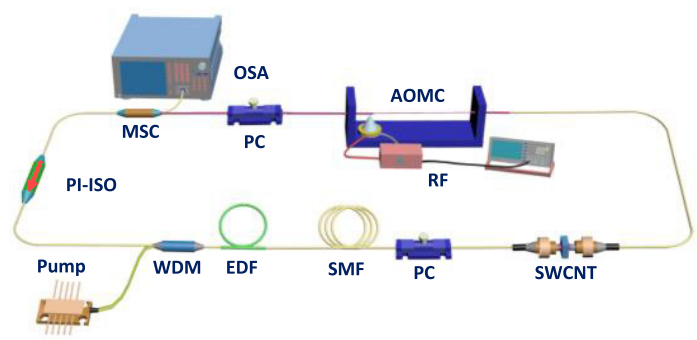

B

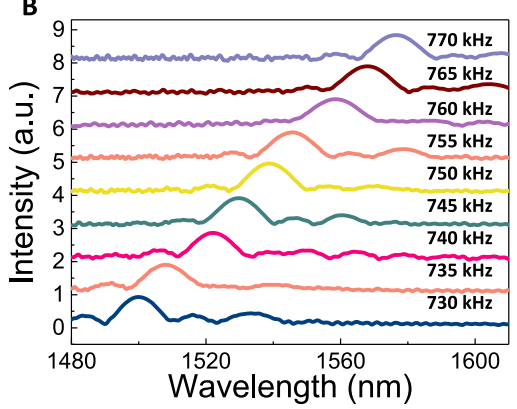

D

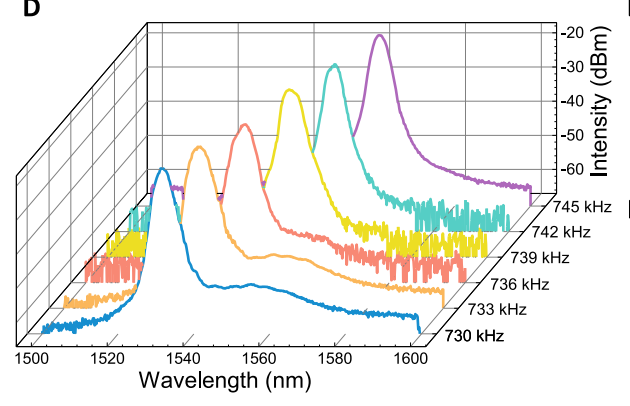

$E$

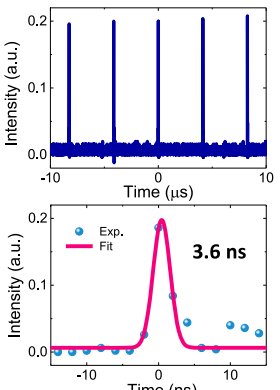

Figure 13: Mode locking (ML) wavelength tunable fiber laser.

(A) Structure of the tunable bandpass filter (TBPF) and modal light in different parts of the TBPF. (B) The transmission spectra of the TBPF at different applied frequencies. (C) The experimental configuration of the tunable ML fiber laser. WDM: wavelength division multiplexer; EDF: erbium-doped fiber; PI-ISO, polarization-independent isolator; SWCNT, single-walled carbon nanotube. (D) The laser spectra with wavelength tunability via controlling the radio frequency (RF) frequency. (E) Pulse trains of the wavelength-tunable ML laser. (F) The measurement of pulse width (Reproduced with permission from Cheng et al. [189]). 
Specifically, the energy oscillation depends on the light wave interactions through nonlinearity, dispersion, intracavity gain and loss and contributes to the ultimate ML formation [202]. Although extensive theoretical literatures on ML dynamics have been investigated, the experiments have more restrictions and are always limited by the measurements only utilizing fast photoelectric detectors (PDs).

Conventional PDs are incapable of capturing ultrafast dynamic process with high resolution and sensitivity due to the restrictions of (1) the narrow electric-bandwidths; (2) the time for reading out the data from sensor arrays and (3) the fundamental compromise between sensitivity and frame rate [203]. The recently developed time-stretched dispersion Fourier transform (TS-DFT) method provides an elegant way for exploring the ultrafast dynamics [204-219], as shown in Table 2.

The experimental demonstrations of real-time observation of spectral evolution dynamics in ML lasers are enabled by controlling the pump switch. Generally, the pump switch is demonstrated by employing a chopper or just turning on the pump laser and is suitable for the observation of ML formation through pump injection. However, pump injection switch is incapable of obtaining the intracavity perturbation and variation of the ML state. Recently the mode switching capability of the dualresonant AOMC has been proved to obtain the real-time observation of intracavity pulse evolution among different ML vortex modes [112].

Figure 14A shows the experimental setup of the narrowlinewidth vortex ML fiber laser. To explore the mode switching dynamic process of the vortex mode switching ML fiber laser, the observation of the vortex mode switching has been demonstrated by the real-time OSC. Figure 14B exhibits the whole process of the pulse evolution during the vortex mode switching. There exist obvious laser strike regions in every vortex mode switching process.
Figure 14C and D depicts the detailed information of interesting instabilities at the laser strike regions of different vortex mode switching processes. When detuned from the stable vortex ML state, the laser experiences a ML collapse phase. As the cavity energy accumulates, there exist large laser spikes, that is, relaxation oscillation. Due to the spatial mode interaction, the laser spikes exhibit irregular fluctuation type rather than conventional raising style. To be specific, the spatial mode competition leads to an energy coupling between different spatial modes. Therefore, the corresponding laser spikes exhibit envelope fluctuation due to the undulant energy accumulation. Vortex mode switching between different order modes experiences a more complex process due to a large interval of effective refractive index, which takes more time to recover the pulse amplitude than that of same order vortex modes.

To further explore the dynamic process of vortex switching, a TS-DFT method has been employed to demonstrate the real-time observation of the single-shot spectrum evolution. Figure 14E shows the experimental result of the wavelength red shift in the vortex mode switching process from $\mathrm{OAM}_{+1}$ mode to $\mathrm{OAM}_{0}$ mode. Firstly, the laser remains a stable ML state at $\mathrm{OAM}_{+1}$ mode. When the RF signal is applied on the AOMC component, the stable ML state of $\mathrm{OAM}_{+1}$ starts to quiet down and collapses to the initial quantum state, which exhibits ultralow power. Afterward there emerge some small laser spikes mainly originated from relaxation oscillation. When the laser cavity energy accumulates enough, a dominant laser spike appears. Then, the lasing wavelength experiences a shifting phase determined by the spatial mode conversion from $\mathrm{OAM}_{+1}$ mode to $\mathrm{OAM}_{0}$ mode. AOMC-based switching strategy provides a higher degree of regulatory freedom and enables the intracavity energy dynamic perturbation. Therefore, it is considered as a new regulatory mechanism to achieve more abundant ML dynamics observations.

Table 2: The dynamics observations in mode locking (ML) fiber lasers via dispersion Fourier transform (DFT) method.

\begin{tabular}{lllll}
\hline Laser type & Switch mode & Method & Dynamics & Ref. \\
\hline ML fiber laser & Pump switch & DFT & Rogue wave formation \\
Soliton fiber laser & Pump switch & DFT & Q-switch and ML build-up \\
DS fiber laser & Pump switch & DFT & DS molecules build-up \\
DS fiber laser & Pump switch & DFT + time lens & DS break-up and collisions \\
Soliton fiber laser & Pump switch & DFT & Soliton explosions & {$[208]$} \\
Soliton fiber laser & Pump switch & DFT & Soliton triplets vibrating \\
Bidirectional soliton laser & Pump switch & DFT & Bidirectional soliton build-up \\
Narrow-linewidth ML laser & AOIs & DFT & Vortex mode switching & {$[218]$} \\
\hline
\end{tabular}


A

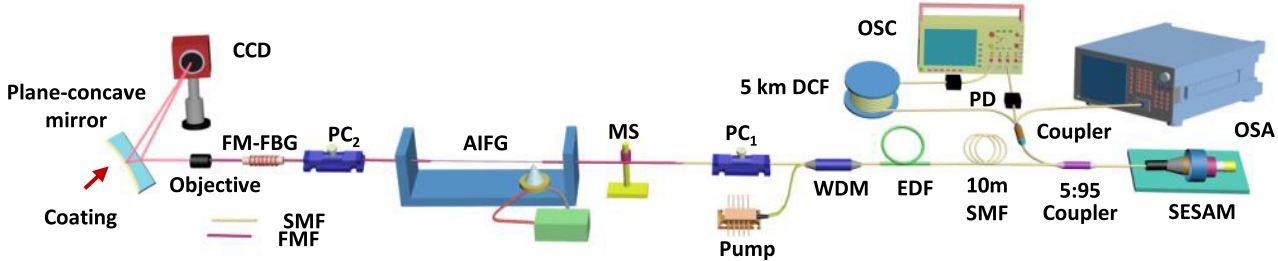

B

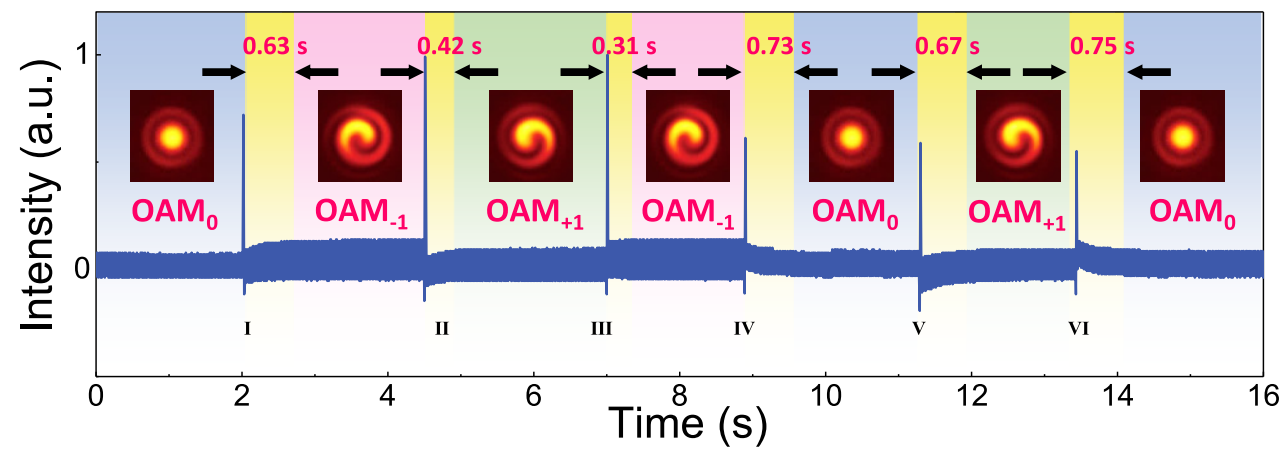

C

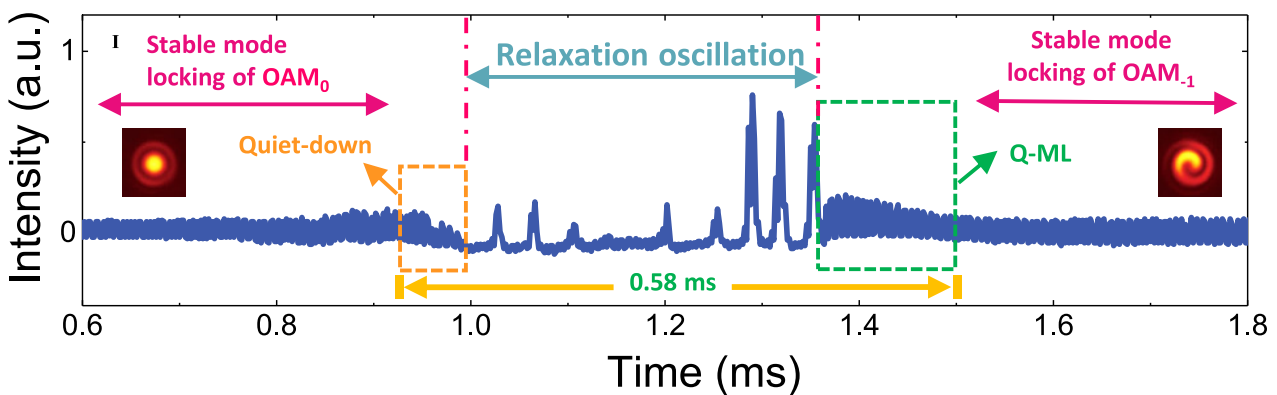

D

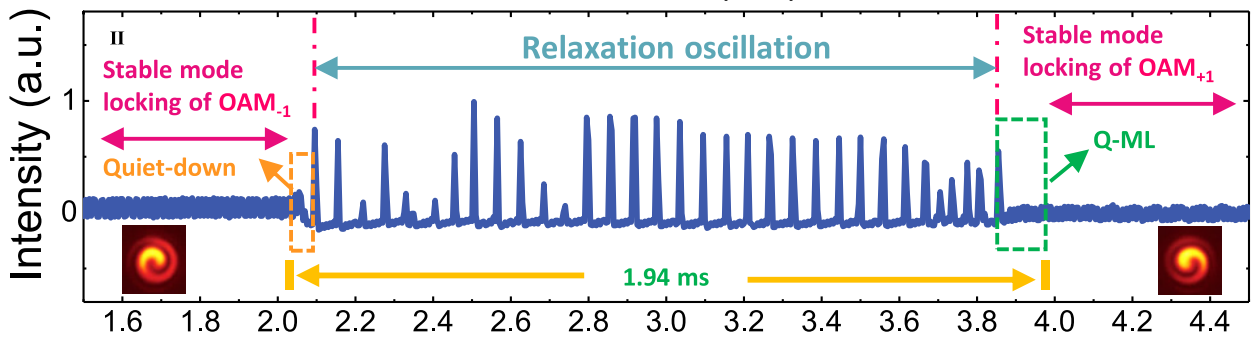

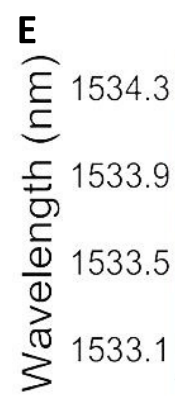

Time (ms)

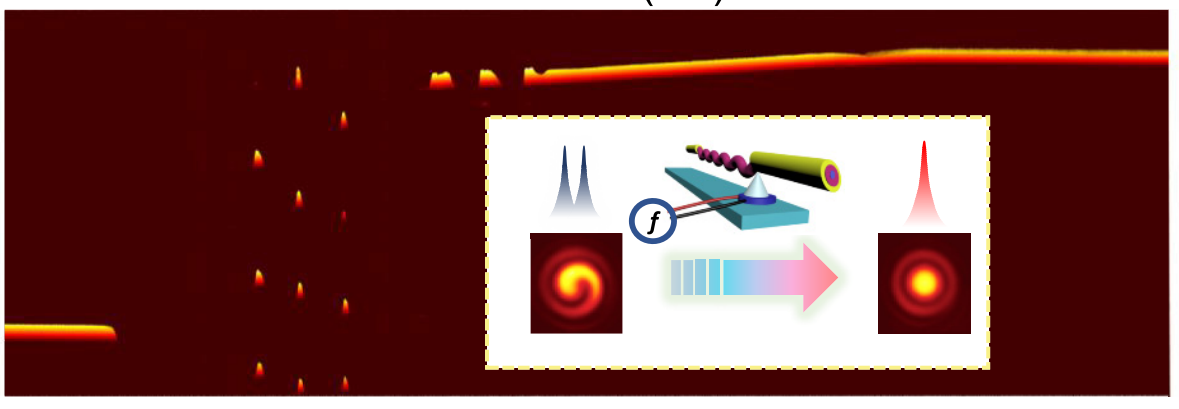

0

1000

2000

3000

4000

5000

6000

7000

Roundtrip

Figure 14: The experimental setup and real-time observation of vortex mode switching in a mode-locked fiber laser.

(A) The schematic diagram of vortex mode switching fiber laser setup. OSA: optical spectrum analyzer; PC: polarization controller; MS: mode stripper. (B) The real-time information of mode switching dynamic processes among three vortex modes $\left(O A M_{0}, O_{-1} M_{-1}\right.$ and $\left.O A M_{+1}\right)$. The detailed information of the strike regions named as I (C) and II (D). (E) The whole spectrum evolution of vortex mode switching dynamics obtained by time-stretched dispersion Fourier transform (TS-DFT) technique (Reproduced with permission from Lu et al. [112]). 


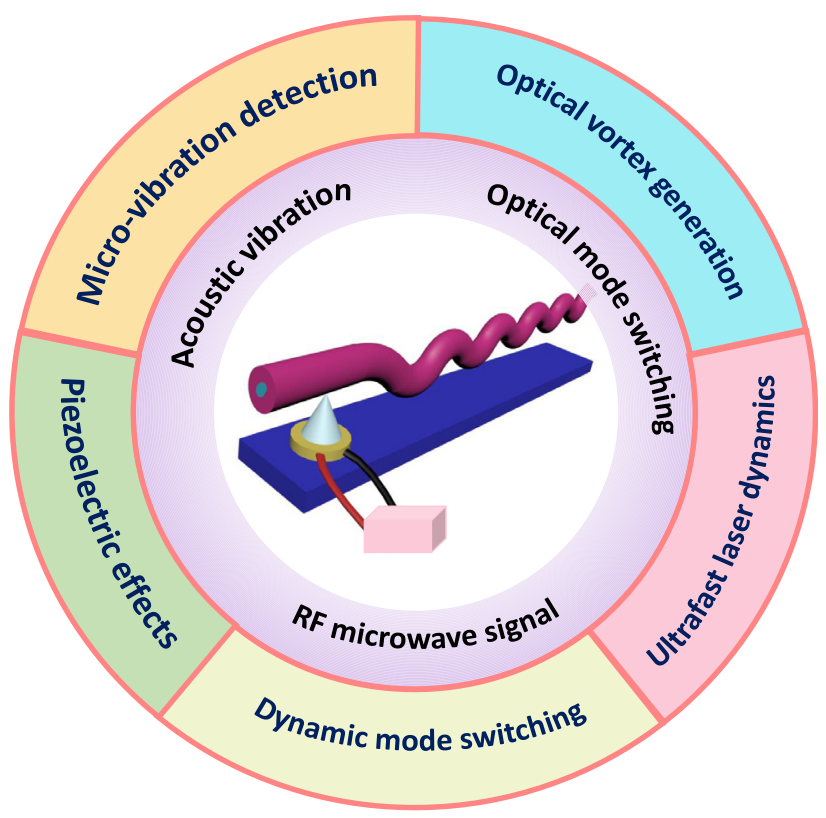

Figure 15: The academic and engineering applications of acoustooptic mode converters (AOMCs).

\section{Conclusions and perspectives}

Toward spatial mode conversion, various investigations of AOIs in FMFs have constructed the dynamic control by microwave RF signal modulation, which provides a new degree of freedom in HOMs and OAMs generation. Benefiting from the dynamic tunability of AOMCs, switching topological charges of OAM modes, heterodyne detections, HOM fiber lasers, Brillouin lasers, ultrafast tunable lasers as well as ML dynamics are summarized in this review with exhibition of Figure 15. To date, dynamic fiber gratings based on the AOI has been the intensive research hotspot in recent years. It involves that how to achieve the resonant response spectrum from narrow bandwidth to wideband conversion, from single resonance of mode conversion to dual resonances with mode switching, from low efficiency to deep resonant peaks via cascading AOMCs with the development of AOMCs.

However, what is the future of the AOMC? How to explore the new mechanisms and practical applications of the AOI in optical fibers? Compared to static mode converters of other schemes, AOIs take advantages of the intriguing properties of fast switching and frequency shifting. The dynamic mode manipulation based on AOMCs exhibits promising values in future researches of AOIs in optical fibers.

This review is aimed to discuss and excite the further interest of the AOI in fibers. Potential research topics of AOMCs include as follows:
(1) AOIs are enabled by applying microwave RF signals on the PZTs for controlling the light propagation. Note that the microwave signal also travels along the optical fibers with the modulation of the light wave. The microwave signal that carries information may be demodulated from the output light wave signal. Moreover, operating microwave signal on the transducer in a soliton ML cavity enhances the nonlinear interactions between microwave signal and ultrashort soliton pulses. There may emerge microwave spectrum purifying for generating high-quality microwave signal toward various applications. The underlying mechanism of nonlinear interactions between microwave signal and optical solitons on both the time scale and spatial scale also attracts intensive attentions and will open a new future of AOIs.

(2) The dynamic evolutions of AO modulation in fibers lead to a new way for figuring out the effect of AOIs, especially in ultrafast ML lasers. Thanks to the development of TS-DFT technique, ultrafast dynamic process can be observed from a real-time view. ML process exhibits complex mechanism from quantum initial state to ultimate ML state. From now on, researchers have investigated theoretically and experimentally on fundamental mode ML dynamics. However, multiple spatial modes interact in the ML laser cavity show more interesting phenomena in the ML process. By introducing the dynamic control of spatial mode conversion, AOMCs can provide new possibilities on revealing the spatial mode evolution mechanism (even spatiotemporal ML) in HOM ML lasers.

(3) Vector mode field has attracted numerous attentions due to its intriguing properties of donut-type intensity profiles and unique polarization distributions. AOMCs possess the flexibility of mode switching, which provides a high degree of freedom in light manipulation. For example, the STED system requires a fundamental mode source for stimulation and a vector mode source for depletion. The AOMC is capable of the integration for stimulation and depletion sources and contributes to super-diffraction imaging. Optical tweezers incorporating OAM modes lead to particle manipulation including optical catching, moving and even rotation. AOMCs provide the fast switch of OAMs with different topological charges, which can lead to high degree of flexibility in microparticle operations. For example, the switch of OAM light between " +1 order" and " -1 order" may control the particle to rotate between clockwise and counterclockwise.

(4) From the practical point of view, AOIs can control the mode conversion with different conversion efficiencies, 
which can be utilized in device tests. For example, FM-FBG is now a very useful fiber device in HOM fiber optics, especially employed in fiber laser configurations. Conventionally, only reflecting wavelengths of different mode resonances in an FM-FBG are measured spectrally by using an off-set technique. The off-set technique only ensures that HOMs are generated but the proportions of each HOM are out of control. However, the unknown reflectivity of each spatial mode makes FM-FBGs restricted to broad applications. AOMC has the possibility of generating certain proportion of multiHOMs by controlling the mode conversion efficiency dynamically. The controllable HOM source is promising for characterizing the reflection efficiencies of the FM-FBG component.

(5) AOMC has been proved to be employed in high power laser fabrications. Conventional in-line fiber devices like LPGs and MSCs depend on the inner refractive index modulation or evanescent field coupling, which are incapable of resisting high temperature created by highpower laser propagation in the fiber. However, AOIs exploit external fiber vibration to control the light propagation without any physical damage of the optical fiber. Therefore, AOMC is quite promising in high-power laser science with agile mode switching capability.

However, the AOIs in optical fibers also have the following challenges:

(1) AO effects exploit acoustic vibrations on optical fibers, which introduces vibration perturbations in the fiber system. The dynamic tunability of light-ultrasound interaction also introduces dynamic variation characteristics in an AOMC. Compared to passive components such as LPGs and MSCs, AOMCs have more complexity in practical uses. Obviously, the environmental vibrations (close to the applied vibration on the AOMC) may disorder the acoustic wave and decrease the mode conversion efficiency.

(2) AOMC device, as an active modulator, requires a modulation unit of modulation signal (microwave RF wave, usually company with a voltage amplifier) to provide the active control. These additional parts may limit the device size for the integration of the fiber system. Besides, according to the theory of the AOIs in fibers, a highly efficient mode conversion needs a several-centimeter length of the AOI region. To reduce the size of the AOMC, one straightforward way is to decline the fiber diameter by HF etching method. With a small diameter of fiber cladding, the vibration efficiency will be improved so that the mode conversion requires short coupling region length. However, decreasing the fiber diameter also reduces the robustness of the AOMC device.

Indeed, the mechanisms and applications of AOIs in optical fibers, especially in special fibers such as FMFs, MMFs, PCFs, ring-core fibers, rare-earth-doped fibers, double-cladding fibers, still require further explorations, not limited to the above suggestions. It is believed that the transformation of scientific researches of AOIs in optical fibers into industrial applications will benefit the future fiber optics.

Acknowledgments: Xianglong Zeng acknowledges the Program for Professor of Special Appointment (Eastern Scholar) at Shanghai Institutions of Higher Learning.

Author contribution: All the authors have accepted responsibility for the entire content of this submitted manuscript and approved submission.

Research funding: National Natural Science Foundation of China (NSFC) (91750108, 61635006); Science and Tech-nology Commission of Shanghai Municipality (STCSM) (20JC1415700, 16520720900); 111 Project (D20031); National Key Research and Development Program of China (2018YFB1801800).

Conflict of interest statement: The authors declare no conflicts of interest regarding this article.

\section{References}

[1] A. M. Yao and M. J. Padgett, "Orbital angular momentum: origins, behavior and applications," Adv. Opt. Photon., vol. 3, no. 2, pp. 161-204, 2011.

[2] G. C. G. Berkhout, M. P. J. Lavery, J. Courtial, M. W. Beijersbergen, and M. J. Padgett, "Efficient sorting of orbital angular momentum states of light,” Phys. Rev. Lett., vol. 105, no. 15, p. 153601, 2010.

[3] J. Wang and A. E. Willner, "Twisted communications using orbital angular momentum (tutorial talk)," in Optical Fiber Communication Conference, paper Th1H.5, 2016.

[4] R. van Uden, R. Correa, E. Lopez, et al., “Ultra-high-density spatial division multiplexing with a few-mode multicore fibre," Nat. Photonics, vol. 8, no. 11, pp. 865-870, 2014.

[5] D. J. Richardson, J. M. Fini, and L. E. Nelson, "Space-division multiplexing in optical fibres," Nat. Photonics, vol. 7, no. 5, pp. 354-362, 2013.

[6] H. R. Stuart, "Dispersive multiplexing in multimode optical fiber," Science, vol. 289, pp. 281-283, 2000.

[7] P. J. Winzer, “Energy-efficient optical transport capacity scaling through spatial multiplexing," IEEE Photon. Technol. Lett., vol. 23, no. 13, pp. 851-853, 2011.

[8] P. M. Krummrich, “Optical amplification and optical filter based signal processing for cost and energy efficient spatial multiplexing," Opt. Express, vol. 19, no. 17, pp. 16636-16652, 2011.

[9] M. Padgett and R. Bowman, "Tweezers with a twist," Nat. Photonics, vol. 5, no. 6, pp. 343-348, 2011. 
[10] D. G. Grier, “A revolution in optical manipulation," Nature, vol. 424, pp. 810-816, 2003.

[11] Y. Shen, X. Wang, Z. Xie, et al., "Optical vortices 30 years on: OAM manipulation from topological charge to multiple singularities," Light Sci. Appl., vol. 8, no. 1, p. 90, 2019.

[12] S. Furhapter, A. Jesacher, S. Bernet, and M. Ritsch-Marte, "Spiral interferometry," Opt. Lett., vol. 30, no. 15, pp. 1953-1955, 2005.

[13] S. Furhapter, A. Jesacher, S. Bernet, and M. Ritsch-Marte, "Spiral phase contrast imaging in microscopy," Opt. Express, vol. 13, no. 3, pp. 689-694, 2005.

[14] L. Torner, J. Torres, and S. Carrasco, "Digital spiral imaging," Opt. Express, vol. 13, no. 3, pp. 873-881, 2005.

[15] S. Bernet, A. Jesacher, S. Furhapter, C. Maurer, and M. RitschMarte, "Quantitative imaging of complex samples by spiral phase contrast microscopy,” Opt. Express, vol. 14, no. 9, pp. 3792-3805, 2006.

[16] B. Jack, J. Leach, J. Romero, et al., "Holographic ghost imaging and the violation of a Bell inequality," Phys. Rev. Lett., vol. 103, no. 8, p. 083602, 2009.

[17] S. Oemrawsingh, X. Ma, D. Voigt, et al., "Experimental demonstration of fractional orbital angular momentum entanglement of two photons," Phys. Rev. Lett., vol. 95, no. 24, p. 240501, 2005.

[18] J. Leach, B. Jack, J. Romero, et al., "Quantum correlations in optical angle-orbital angular momentum variables," Science, vol. 329, pp. 662-665, 2010.

[19] A. Vaziri, G. Weihs, and A. Zeilinger, "Experimental two-photon, three-dimensional entanglement for quantum communication," Phys. Rev. Lett., vol. 89, no. 24, p. 240401, 2002.

[20] E. Nagali, F. Sciarrino, F. D. Martini, et al., "Quantum information transfer from spin to orbital angular momentum of photons," Phys. Rev. Lett., vol. 103, no. 1, p. 013601, 2009.

[21] K. Toyoda, K. Miyamoto, N. Aoki, R. Morita, and T. Omatsu, "Using optical vortex to control the chirality of twisted metal nanostructures," Nano Lett., vol. 12, no. 7, pp. 3645-3649, 2012.

[22] F. Takahashi, K. Miyamoto, H. Hidai, K. Yamane, R. Morita, and T. Omatsu, "Picosecond optical vortex pulse illumination forms a monocrystalline silicon needle,” Sci. Rep., vol. 6, p. 21738, 2016.

[23] J. Ni, C. Wang, C. Zhang, et al., "Three-dimensional chiral microstructures fabricated by structured optical vortices in isotropic material," Light Sci. Appl., vol. 6, p. e17011, 2017.

[24] D. L. Andrews, "Quantum formulation for nanoscale optical and material chirality: symmetry issues, space and time parity, and observables," J. Opt., vol. 20, no. 3, p. 033003, 2018.

[25] J. Wang, J. Y. Yang, I. M. Fazal, et al., "Terabit free-space data transmission employing orbital angular momentum multiplexing," Nat. Photonics, vol. 6, no. 7, pp. 488-496, 2012.

[26] B. Ndagano, I. Nape, M. A. Cox, C. Rosales-Guzman, and A. Forbes, "Creation and detection of vector vortex modes for classical and quantum communication," J. Lightwave Technol., vol. 36, no. 2, pp. 292-301, 2018.

[27] L. Gong, Q. Zhao, H. Zhang, et al., "Optical orbital-angularmomentum-multiplexed data transmission under high scattering," Light Sci. Appl., vol. 8, p. 27, 2019.

[28] Z. Xie, S. Gao, T. Lei, et al., "Integrated (de)multiplexer for orbital angular momentum fiber communication," Photon. Res., vol. 6, no. 7, pp. 743-749, 2018.

[29] T. Wang, F. Wang, F. Shi, et al., "Generation of femtosecond optical vortex beams in all-fiber mode-locked fiber laser using mode selective coupler," J. Lightwave Technol., vol. 35, no. 11, pp. 2161-2166, 2017.

[30] S. Yao, G. Ren, Y. Shen, Y. Jiang, and S. Jian, "Tunable orbital angular momentum generation using all-fiber fused coupler," IEEE Photon. Technol. Lett., vol. 30, no. 1, pp. 99-102, 2017.

[31] H. Wan, J. Wang, Z. Zhang, J. Wang, S. Ruan, and L. Zhang, "Passively mode-locked Ytterbium-doped fiber laser with cylindrical vector beam generation based on mode selective coupler," J. Lightwave Technol., vol. 36, no. 16, pp. 3403-3407, 2018.

[32] Z. S. Eznaveh, J. C. A. Zacarias, J. E. A. Lopez, et al., "Photonic lantern broadband orbital angular momentum mode multiplexer," Opt. Express, vol. 26, no. 23, pp. 30042-30051, 2018.

[33] X. Sai, Y. Li, C. Yang, et al., "Design of elliptical-core modeselective photonic lanterns with six modes for MIMO-free mode division multiplexing systems," Opt. Lett., vol. 42, no. 21, pp. 4355-4358, 2017.

[34] S. G. Leon-Saval, N. K. Fontaine, J. R. Salazar-Gil, B. Ercan, R. Ryf, and J. Bland-Hawthorn, "Mode-selective photonic lanterns for space-division multiplexing," Opt. Express, vol. 22, no. 1, pp. 1036-1044, 2014.

[35] Y. Zhao, Y. Liu, L. Zhang, C. Zhang, J. Wen, and T. Wang, “Mode converter based on the long-period fiber gratings written in the two-mode fiber," Opt. Express, vol. 24, no. 6, pp. 6186-6195, 2016.

[36] Y. Zhao, Z. Liu, Y. Liu, C. Mou, T. Wang, and Y. Yang, "Ultrabroadband fiber mode converter based on apodized phaseshifted long-period gratings," Opt. Lett., vol. 44, no. 24 , pp. 5905-5908, 2019.

[37] C. Fu, S. Liu, Z. Bai, et al., "Orbital angular momentum mode converter based on helical long period fiber grating inscribed by hydrogen-oxygen flame," J. Lightwave Technol., vol. 36, no. 9, pp. 1683-1688, 2018.

[38] Y. Han, Y. Liu, Z. Wang, et al.., “Controllable all-fiber generation/ conversion of circularly polarized orbital angular momentum beams using long period fiber gratings," Nanophotonics, vol. 7, no. 1, pp. 287-293, 2017.

[39] L. Wang, P. Vaity, B. Ung, Y. Messaddeq, L. A. Rusch, and S. LaRochelle, "Characterization of OAM fibers using fiber Bragg gratings,” Opt. Express, vol. 22, no. 13, pp. 15653-15661, 2014.

[40] Z. Lin, A. Wang, L. Xu, et al., "Generation of optical vortices using a helical fiber Bragg grating," J. Lightwave Technol., vol. 32, no. 11, pp. 2152-2156, 2014.

[41] Y. Mi, H. Li, and G. Ren, "Vector mode conversion based on an asymmetric fiber Bragg grating in few-mode fibers," Appl. Opt., vol. 56, no. 25, pp. 7305-7310, 2017.

[42] H. Yao, F. Shi, Z. Wu, et al., "A mode generator and multiplexer at visible wavelength based on all-fiber mode selective coupler," Nanophotonics, vol. 9, no. 4, pp. 973-981, 2020.

[43] B. Y. Kim, J. N. Blake, H. E. Engan, and H. J. Shaw, "All-fiber acousto-optic frequency shifter," Opt. Lett., vol. 11, no. 6, pp. 389-391, 1986.

[44] A. A. P. Pohl, R. A. Oliveira, R. E. D. Silva, et al., "Advances and new applications using the acousto-optic effect in optical fibers," Photonic Sens., vol. 3, no. 1, pp. 1-25, 2013.

[45] H. E. Engan and B. Y. Kim, "Propagation and optical interaction of guided acoustic waves in two-mode optical fibers," J. Lightwave Technol., vol. 6, no. 3, pp. 428-436, 1988. 
[46] W. Huang, C. Xu, and S. K. Chaudhuri, "Modelling and analysis of fiber-optic mode transducers: single fiber with periodic perturbations," J. Lightwave Technol., vol. 9, no. 11, pp. 1431-1438, 1991.

[47] B. Langli, D. Ostling, and K. Bløtekjær, "Axial variations in the acoustooptic phase-mismatch coefficient of two-mode fibers," J. Lightwave Technol., vol. 16, no. 12, pp. 2443-2450, 1998.

[48] C. N. Alexeyev, E. V. Barshak, A. V. Volyar, and M. A. Yavorsky, "Perturbation theory approach for the wave equation in fibre acousto-optics," J. Opt., vol. 12, no. 11, p. 115708, 2010.

[49] M. W. Haakestad and J. Skaar, "Slow and fast light in optical fibers using acoustooptic coupling between two copropagating modes," Opt. Express, vol. 17, no. 1, pp. 346-357, 2009.

[50] M. A. Yavorsky, D. V. Vikulin, E. V. Barshak, B. P. Lapin, and C. N. Alexeyev, "Revised model of acousto-optic interaction in optical fibers endowed with a flexural wave," Opt. Lett., vol. 44, no. 3, pp. 598-601, 2019.

[51] D. Ostling and H. E. Engan, "Spectral flattening by an all-fiber acousto-optic tunable filter," IEEE Int. Ultrason. Symp., vol. 2, no. 2, pp. 837-840, 1995.

[52] D. Ostling and H. E. Engan, "Acousto-optic tunable filters in twomode fibers,” Opt. Fiber Technol., vol. 3, no. 2, pp. 177-183, 1997.

[53] H. S. Kim, S. H. Yun, I. K. Kwang, and B. Y. Kim, "All-fiber acoustooptic tunable notch filter with electronically controllable spectral profile," Opt. Lett., vol. 22, no. 19, pp. 1476-1478, 1997.

[54] D. O. Culverhouse, S. H. Yun, D. J. Richardson, et al., "Low-loss all-fiber acousto-optic tunable filter," Opt. Lett., vol. 22, no. 2, pp. 96-98, 1997.

[55] I. K. Hwang, S. H. Yun, and B. Y. Kim, "All-fiber tunable comb filter with nonreciprocal transmission," IEEE Photon. Technol. Lett., vol. 10, no. 10, pp. 1437-1439, 1998.

[56] R. Feced, C. Alegria, M. N. Zervas, and R. I. Laming, "Acoustooptic attenuation filters based on tapered optical fibers," IEEE J. Sel. Top. Quantum Elec., vol. 5, no. 5, pp. 1278-1288, 1999.

[57] A. Diez, G. Kakarantzas, T. A. Birks, and P. S. J. Russell, "Acoustic stop-bands in periodically microtapered optical fibers," Appl. Phys. Lett., vol. 76, no. 23, pp. 3481-3483, 2000.

[58] D. A. Satorius, T. E. Dimmick, and G. L. Burdge, "Double-pass acoustooptic tunable bandpass filter with zero frequency shift and reduced polarization sensitivity," IEEE Photon. Technol. Lett., vol. 14, no. 9, pp. 1324-1326, 2002.

[59] W. Zhang, F. Gao, F. Bo, Q. Wu, G. Zhang, and J. Xu, "All-fiber acousto-optic tunable notch filter with a fiber winding driven by a cuneal acoustic transducer," Opt. Lett., vol. 36, no. 2 , pp. 271-273, 2011

[60] W. Zhang, L. Huang, F. Gao, F. Bo, G. Zhang, and J. Xu, "All-fiber tunable Mach-Zehnder interferometer based on an acoustooptic tunable filter cascaded with a tapered fiber," Opt. Commun., vol. 292, pp. 46-48, 2013.

[61] W. Zhang, L. Huang, F. Gao, F. Bo, G. Zhang, and J. Xu, "Tunable broadband light coupler based on two parallel all-fiber acoustooptic tunable filters," Opt. Express, vol. 21, no. 14, pp. 16621-16628, 2013.

[62] H. Zhang, S. Kang, B. Liu, H. Dong, and Y. Miao, "All-fiber acousto-optic tunable bandpass filter based on a lateral offset fiber splicing structure," IEEE Photon. J., vol. 7, no. 1, p. 2700312, 2015.
[63] G. R. Melendez, M. Bello-Jimenez, O. Pottiez, and M. Andres, "Improved all-fiber acousto-optic tunable bandpass filter," IEEE Photon. Technol. Lett., vol. 29, no. 12, pp. 1015-1018, 2017.

[64] L. Huang, W. Zhang, Y. Li, et al., "Acousto-optic tunable bandpass filter based on acoustic-flexural-wave-induced fiber birefringence," Opt. Lett., vol. 43, no. 21, pp. 5431-5434, 2018.

[65] W. F. Liu, P. S. J. Russell, and L. Dong, "Acousto-optic superlattice modulator using a fiber Bragg grating," Opt. Lett., vol. 22, no. 19, pp. 1515-1517, 1997.

[66] W. F. Liu, P. S. J. Russell, and L. Dong, " $100 \%$ efficient narrowband acoustooptic tunable reflector using fiber Bragg grating," J. Lightwave Technol., vol. 16, no. 11, pp. 2006-2009, 1998.

[67] P. S. J. Russell and W. F. Liu, "Acousto-optic superlattice modulation in fiber Bragg gratings," J. Opt. Soc. Am. A, vol. 17, no. 8, pp. 1421-1429, 2000.

[68] W. Liu, I. Liu, L. Chung, D. Huang, and C. C. Yang, "Acousticinduced switching of the reflection wavelength in a fiber Bragg grating," Opt. Lett., vol. 25, no. 18, pp. 1319-1321, 2000.

[69] N. Sun, C. Chou, M. Chang, et al.., "Analysis of phase-matching conditions in flexural-wave modulated fiber Bragg grating," J. Lightwave Technol., vol. 20, no. 2, pp. 311-315, 2002.

[70] W. J. Lee, B. K. Kim, K. H. Han, and B. Y. Kim, “Dual heterodyne polarization diversity demodulation for fiber-optic interferometers," IEEE Photon. Technol. Lett., vol. 11, no. 9, pp. 1156-1158, 1999.

[71] H. M. Chan, R. Huang, F. Alhassen, et al., "A compact all-fiber LPG-AOTF frequency shifter on single-mode fiber and its application to vibration measurement," IEEE Photon. Technol. Lett., vol. 20, no. 18, pp. 1572-1574, 2008.

[72] W. Zhang, W. Gao, L. Huang, et al., “Optical heterodyne microvibration measurement based on all-fiber acousto-optic frequency shifter," Opt. Express, vol. 23, no. 13, pp. 17576-17583, 2015.

[73] W. Zhang, Z. Chen, B. Jiang, et al., “Optical heterodyne microvibration detection based on all-fiber acousto-optic superlattice modulation," J. Lightwave Technol., vol. 35, no. 18, pp. 3821-3824, 2017.

[74] A. Diez, T. A. Birks, W. H. Reeves, B. J. Mangan, and P. S. J. Russell, "Excitation of cladding modes in photonic crystal fibers by flexural acoustic waves," Opt. Lett., vol. 25, no. 20, pp. 1499-1501, 2000.

[75] M. W. Haakestad and H. E. Engan, "Acoustooptic properties of a weakly multimode solid core photonic crystal fiber," J. Lightwave Technol., vol. 24, no. 2, pp. 838-845, 2006.

[76] M. W. Haakestad and H. E. Engan, "Acoustooptic characterization of a birefringent two-mode photonic crystal fiber," Opt. Express, vol. 14, no. 16, pp. 7319-7328, 2006.

[77] K. S. Hong, H. C. Park, B. Y. Kim, et al., " $1000 \mathrm{~nm}$ tunable acoustooptic filter based on photonic crystal fiber," Appl. Phys. Lett., vol. 92, no. 3, p. 031110, 2008.

[78] M. S. Kang, M. S. Lee, J. C. Yong, and B. Y. Kim, "Characterization of wavelength-tunable single-frequency fiber laser employing acoustooptic tunable filter," J. Lightwave Technol., vol. 24, no. 4, pp. 1812-1823, 2006.

[79] L. Huang, P. Chang, X. Song, et al., "Tunable in-fiber MachZehnder interferometer driven by unique acoustic transducer and its application in tunable multi-wavelength laser," Opt. Express, vol. 24, no. 3, pp. 2406-2412, 2016. 
[80] L. Huang, X. Song, P. Chang, et al., "All-fiber tunable laser based on an acousto-optic tunable filter and a tapered fiber," Opt. Express, vol. 24, no. 7, pp. 7449-7455, 2016.

[81] N. Yan, X. Han, P. Chang, et al., "Tunable dual-wavelength fiber laser with unique gain system based on in-fiber acousto-optic Mach-Zehnder interferometer," Opt. Express, vol. 25, no. 22, pp. 27609-27615, 2017.

[82] E. H. Escobar, M. B. Jiménez, A. C. Avilés, et al., "Experimental study of an in-fiber acousto-optic tunable bandpass filter for single- and dual-wavelength operation in a thulium-doped fiber laser," Opt. Express, vol. 27, no. 26, pp. 38602-38613, 2019.

[83] D. Zalvidea, N. A. Russo, R. Duchowicz, et al., "High-repetition rate acoustic-induced q-switched all-fiber laser," Opt. Commun., vol. 244, no. 1-6, pp. 315-319, 2005.

[84] M. Delgado-Pinar, D. Zalvidea, A. Diez, P. Perez-Millan, and M. V. Andrés, "Q-switching of an all-fiber laser by acousto-optic modulation of a fiber Bragg grating," Opt. Express, vol. 14, no. 3, pp. 1106-1112, 2006.

[85] C. Cuadrado-Laborde, M. Delgado-Pinar, S. Torres-Peiró, A. Díez, and M. V. Andrés, "Q-switched all-fibre laser using a fibre-optic resonant acousto-optic modulator," Opt. Commun., vol. 274, no. 2, pp. 407-411, 2007.

[86] C. Cuadrado-Laborde, A. Diez, M. Delgado-Pinar, J. L. Cruz, and M. V. Andrés, "Mode locking of an all-fiber laser by acousto-optic superlattice modulation," Opt. Lett., vol. 34, no. 7, pp. 1111-1113, 2009.

[87] C. Cuadrado-Laborde, A. Díez, J. L. Cruz, and M. V. Andrés, "Experimental study of an all-fiber laser actively mode-locked by standing-wave acousto-optic modulation," Appl. Phys. B, vol. 99, no. 1-2, pp. 95-99, 2010.

[88] M. Bello-Jiménez, C. Cuadrado-Laborde, A. Diez, J. L. Cruz, and M. V. Andrés, "Experimental study of an actively mode-locked fiber ring laser based on in-fiber amplitude modulation," Appl. Phys. B, vol. 105, no. 2, pp. 269-276, 2011.

[89] C. Cuadrado-Laborde, A. Diez, J. L. Cruz, and M. V. Andrés, "Q-switched and modelocked all-fiber lasers based on advanced acousto-optic devices," Laser Photon. Rev., vol. 5, no. 3, pp. 404-421, 2011.

[90] M. Bello-Jimenez, C. Cuadrado-Laborde, A. Diez, J. L. Cruz, M. V. Andres, and A. Rodriguez-Cobos, "Mode-locked all-fiber ring laser based on broad bandwidth in-fiber acousto-optic modulator," Appl. Phys. B, vol. 110, pp. 73-80, 2013.

[91] W. Zhang, K. Wei, H. Wang, et al., "Tunable-wavelength picosecond vortex generation in fiber and its application in frequency-doubled vortex," J. Opt., vol. 20, no. 1, p. 014004, 2018.

[92] Y. Li, L. Huang, H. Han, et al., “Acousto-optic tunable ultrafast laser with vector-mode-coupling-induced polarization conversion," Photon. Res., vol. 7, no. 7, pp. 798-805, 2019.

[93] P. Z. Dashti, F. Alhassen, and H. P. Lee, "Observation of orbital angular momentum transfer between acoustic and optical vortices in optical fiber," Phys. Rev. Lett., vol. 96, no. 4, p. 043604, 2006.

[94] W. Zhang, L. Huang, K. Wei, et al., “Cylindrical vector beam generation in fiber with mode selectivity and wavelength tunability over broadband by acoustic flexural wave," Opt. Express, vol. 24, no. 10, pp. 10376-10384, 2016.

[95] W. Zhang, K. Wei, L. Huang, et al., "Optical vortex generation with wavelength tunability based on an acoustically-induced fiber grating," Opt. Express, vol. 24, no. 17, pp. 19278-19285, 2016.

[96] K. Wei, W. Zhang, L. Huang, et al., "Generation of cylindrical vector beams and optical vortex by two acoustically induced fiber gratings with orthogonal vibration directions," Opt. Express, vol. 25, no. 3, pp. 2733-2741, 2017.

[97] L. Carrión-Higueras, E. P. Alcusa-Sáez, A. Díez, and M. V. Andrés, "All-fiber laser with intracavity acousto-optic dynamic mode converter for efficient generation of radially polarized cylindrical vector beams," IEEE Photon. J., vol. 9, no. 1, p. 1500507, 2017.

[98] T. A. Birks, D. O. Culverhouse, S. G. Farwell, P. St, and J. Russell, “2 2 2 Single-mode fiber routing switch," Opt. Lett., vol. 21, no. 10, pp. 722-724, 1996.

[99] H. S. Park, K. Y. Song, and S. H. Yun, “All-fiber wavelengthtunable acoustooptic switches based on intermodal coupling in fibers," J. Lightwave Technol., vol. 20, no. 10, pp. 1864-1868, 2002.

[100] I. K. Hwang, S. H. Yun, and B. Y. Kim, "All-fiber-optic nonreciprocal modulator," Opt. Lett., vol. 22, no. 8, pp. 507-509, 1997.

[101] M. Berwick and D. A. Jackson, "Coaxial optical-fiber frequency shifter," Opt. Lett., vol. 17, pp. 270-272, 1992.

[102] T. A. Birks, S. G. Farwell, P. St. J. Russell, and C. N. Pannell, "Four-port fiber frequency shifter with a null taper coupler," Opt. Lett., vol. 19, no. 23, pp. 1964-1966, 1994.

[103] D. O. Culverhouse, T. A. Birks, S. G. Farwell, J. Ward, P. St. J. Russell, " $40-\mathrm{MHz}$ all-fiber acoustooptic frequency shifter," IEEE Photon. Technol. Lett., vol. 8, no. 12, pp. 1636-1637, 1996.

[104] S. H. Yun, B. K. Kim, H. J. Jeong, and B. Y. Kim, "Suppression of polarization dependence in a two-mode-fiber acousto-optic device," Opt. Lett., vol. 21, no. 12, pp. 908-910, 1996.

[105] J. Lu, L. Meng, F. Shi, et al., "Dynamic mode-switchable optical vortex beams using acousto-optic mode converter," Opt. Lett., vol. 43, no. 23, pp. 5841-5844, 2018.

[106] J. Lu, L. Meng, F. Shi, and X. Zeng, “A mode-locked fiber laser with switchable high-order modes using intracavity acoustooptic mode converter," in Optical Fiber Communication Conference, paper W3C.3, 2019.

[107] L. Meng, J. Lu, F. Shi, et al., "Multi-orthogonal high-order mode converter based on acoustically induced fiber gratings," IEEE Photon. Technol. Lett., vol. 31, no. 13, pp. 819-822, 2020.

[108] F. Shi, J. Lu, L. Meng, et al., "All-fiber method for real-time transverse-mode switching of ultrashort pulse," IEEE Photon. Technol. Lett., vol. 32, no. 2, pp. 97-100, 2020.

[109] H. Wu, J. Lu, L. Huang, X. Zeng, and P. Zhou, "All-fiber laser with agile mode-switching capability through intra-cavity conversion," IEEE Photon. J., vol. 12, no. 2, p. 1500709, 2020.

[110] L. Meng, J. Lu, L. Zhang, F. Shi, and X. Zeng, "Multi-orthogonal high-order modes converter," in Conference on Lasers and Electro-Optics, paper STh3L.4, 2019.

[111] F. Shi, J. Lu, L. Meng, P. Cheng, and X. Zeng, “Delivering transverse-mode switching based on all-fiber femtosecond Laser," in Conference on Lasers and Electro-Optics Europe and European Quantum Electronics, paper cj_11_2, 2019.

[112] J. Lu, F. Shi, L. Meng, et al., "Real-time observation of vortex mode switching in a narrow-linewidth mode-locked fiber laser," Photon. Res., vol. 8, no. 7, pp. 1203-1212, 2020. 
[113] K. J. Lee, H. C. Park, H. S. Park, and B. Y. Kim, "Highly efficient all-fiber tunable polarization filter using torsional acoustic wave," Opt. Express, vol. 15, no. 19, pp. 12362-12367, 2007.

[114] S. D. Lim, H. C. Park, and B. Y. Kim, "Twist effect on spectral properties of two-mode fiber acousto-optic filters," Opt. Express, vol. 16, no. 17, pp. 13042-13051, 2008.

[115] K. J. Lee, I. K. Hwang, H. C. Park, and B. Y. Kim, “Polarizationindependent all-fiber acousto-optic tunable filter using torsional acoustic wave," IEEE Photon. Technol. Lett., vol. 22, no. 8, pp. 523-525, 2010.

[116] J. Ko, K. J. Lee, and B. Y. Kim, "Spectral shaping of an all-fiber torsional acousto-optic tunable filter," Appl. Opt., vol. 53, no. 36, pp. 8499-506, 2014.

[117] D. R. Song, K. J. Lee, and B. Y. Kim, "Band-rejection filtering based on lossy torsional acousto-optic coupling in a single polarization fiber," Opt. Express, vol. 22, no. 20, pp. 24034-24043, 2014

[118] E. P. Alcusa-Sáez, A. Díez, E. Rivera-Pérez, W. Margulis, L. Norin, and M. V. Andrés, "Acousto-optic interaction in polyimide coated optical fibers with flexural waves," Opt. Express, vol. 25, no. 15, pp. 17167-17173, 2017.

[119] R. A. Herrera, C. Hurtado, and P. I. Torres, "Nonlinear acoustooptic coupling in tapered fiber optics: model and experiment," IEEE Photon. Technol. Lett., vol. 29, no. 7, pp. 595-598, 2017.

[120] T. Shoro, H. Kishikawa, and N. Goto, "Analysis of optical OAM mode conversion using elastic vortex wave in graded index optical fiber," Jpn. J. Appl. Phys., vol. 58, p. SGGA04, 2019.

[121] J. N. Blake, B. Y. Kim, H. E. Engan, and H. J. Shaw, "Analysis of intermodal coupling in a two-mode fiber with periodic microbends," Opt. Lett., vol. 12, no. 4, pp. 281-283, 1987.

[122] J. Zhao and X. Liu, "Fiber acousto-optic mode coupling between the higher-order modes with adjacent azimuthal numbers," Opt. Lett., vol. 31, no. 11, pp. 1609-1611, 2006.

[123] J. Zhao, R. Miao, and X. Liu, "Nonresonant cascaded acoustooptic mode coupling,” Opt. Lett., vol. 31, no. 19, pp. 2909-2911, 2006.

[124] T. Pfeifer, L. Gallmann, M. J. Abel, P. M. Nagel, D. M. Neumark, and S. R. Leone, "Heterodyne mixing of laser fields for temporal gating of high-order harmonic generation," Phys. Rev. Lett., vol. 97, no. 16, p. 163901, 2006.

[125] S. Berciaud, L. Cognet, G. A. Blab, and B. Lounis, "Photothermal heterodyne imaging of individual nonfluorescent nanoclusters and nanocrystals," Phys. Rev. Lett., vol. 93, no. 25, p. 257402, 2004.

[126] E. Matsuyama, T. Kondo, H. Oigawa, D. Guo, S. Nemoto, and J. Nakamura, "Principles and application of heterodyne scanning tunnelling spectroscopy," Sci. Rep., vol. 4, p. 6711, 2014.

[127] M. Sasaki, T. Suyama, T. Tanaka, and S. Yokoyama, "Primordial black hole scenario for the gravitational-wave event GW150914," Phys. Rev. Lett., vol. 117, no. 6, p. 061101, 2018.

[128] G. J. Verbiest and M. J. Rost, "Beating beats mixing in heterodyne detection schemes," Nat. Commun., vol. 6, p. 6444 2015.

[129] J. Abbiss and W. Mayo, "Deviation-free Bragg cell frequencyshifting," Appl. Opt., vol. 20, no. 4, pp. 588-553, 1981.

[130] M. Izutsu, S. Shikama, and T. Sueta, "Integrated optical SSB modulator/frequency shifter," IEEE J. Quantum Electron., vol. 17 , no. 11 , pp. 2225-2227, 1981.
[131] Y. Qiu, M. Luo, Z. He, and X. Li, "Balanced detection of 100-Gb/s optical OFDM signals with SSBI cancellation based on fusedtype fiber mode-selective coupler," IEEE Photon. J., vol. 10, no. 5, p. 7105010, 2018.

[132] L. Zhang, J. Lu, L. Meng, et al., "A lower frequency shift based on mode conversion for optical heterodyne micro-vibration measurement," J. Lightwave Technol., vol. 38, no. 21, pp. 6057-6062, 2020.

[133] Y. Xiao, Y. Liu, Z. Wang, X. Liu, and M. Luo, “Design and experimental study of mode selective all-fiber fused mode coupler based on few mode fiber," Acta Phys. Sin., vol. 64, no. 20, p. 204207, 2015.

[134] T. Wang, A. Yang, F. Shi, Y. Huang, J. Wen, and X. Zeng, "Highorder mode lasing in all-FMF laser cavities," Photon. Res., vol. 7, no. 1, pp. 42-49, 2019.

[135] Y. Huang, F. Shi, T. Wang, et al., "High-order mode Yb-doped fiber lasers based on mode selective couplers," Opt. Express, vol. 26, no. 15, pp. 19171-19181, 2018.

[136] W. Zhang, K. Wei, D. Mao, et al., "Generation of femtosecond optical vortex pulse in fiber based on an acoustically induced fiber grating," Opt. Lett., vol. 42, no. 3, pp. 454-457, 2017.

[137] D. Mao, T. Feng, W. Zhang, et al., "Ultrafast all-fiber based cylindrical-vector beam laser," Appl. Phys. Lett., vol. 110, no. 2, p. 021107, 2017.

[138] J. Zheng, A. Yang, T. Wang, et al., "Switchable wavelength vortex beams based on polarization dependent micro-knot resonator," Photon. Res., vol. 6, no. 5, pp. 396-402, 2018.

[139] Z. Zhang, Y. Cai, J. Wang, H. Wan, and L. Zhang, "Switchable dual wavelength cylindrical vector beam generation from a passively mode-locked fiber laser based on carbon nanotubes," IEEE J. Sel. Top. Quantum Electron., vol. 24, no. 3, p. 1100906, 2018.

[140] R. Chen, J. Wang, X. Zhang, et al., "High efficiency all-fiber cylindrical vector beam laser using a long-period fiber grating," Opt. Lett., vol. 43, no. 4, pp. 755-758, 2018.

[141] L. Allen, M. W. Beijersbergen, R. J. C. Spreeuw, and J. P. Woerdman, "Orbital angular momentum of light and the transformation of Laguerre-Gaussian laser modes," Phys. Rev. $A$, vol. 45 , no. 11 , p. $8185,1992$.

[142] S. Ramachandran and P. Kristensen, "Optical vortices in fibers," Nanophotonics, vol. 2, no. 5-6, pp. 455-474, 2013.

[143] Y. Shen, X. Yang, D. Naidoo, X. Fu, and A. Forbes, "Structured ray-wave vector vortex beams in multiple degrees of freedom from a laser," Optica, vol. 7, no. 7, pp. 820-831, 2020.

[144] R. Chen, F. Sun, J. Yao, et al., "Mode locked all-fiber laser generating optical vortex pulses with tunable repetition rate," Appl. Phys. Lett., vol. 112, no. 26, p. 261103, 2018.

[145] Z. Li, J. Peng, Q. Li, Y. Gao, J. Li, and Q. Cao, "Generation of picosecond vortex beam in a self-mode-locked Nd: YVO4 laser," Optoelectron. Lett., vol. 13, no. 3, pp. 188-191, 2017.

[146] Z. Liu, P. Ma, R. Su, et al., "High-power coherent beam polarization combination of fiber lasers: progress and prospect [Invited]," J. Opt. Soc. Am. B, vol. 34, no. 3, pp. A7-A14, 2017.

[147] D. J. Richardson, J. Nilsson, and W. A. Clarkson, "High power fiber lasers: current status and future perspectives [Invited]," J. Opt. Soc. Am. B, vol. 27, no. 11, pp. B63-B92, 2010.

[148] M. N. Zervas and C. A. Codemard, "High power fiber lasers: a review,” IEEE J. Sel. Top. Quantum Electron., vol. 20, no. 5, p. 0904123,2014 
[149] C. Jauregui, J. Limpert, and A. Tünnermann, "High-power fibre lasers," Nat. Photonics, vol. 7, no. 11, pp. 861-867, 2013.

[150] J. Demas, G. Prabhakar, T. He, and S. Ramachandran, "Wavelength-agile high-power sources via four-wave mixing in higher-order fiber modes," Opt. Express, vol. 25, no. 7, pp. 7455-7464, 2017.

[151] Y. J. Huang, P. Y. Chiang, H. C. Liang, K. W. Su, and Y. F. Chen, "High-power q-switched laser with high-order LaguerreGaussian modes: application for extra-cavity harmonic generations," Appl. Phys. B, vol. 105, no. 2, pp. 385-390, 2011.

[152] X. Zhou, Z. Chen, H. Chen, J. Li, and J. Hou, "Mode field adaptation between single-mode fiber and large mode area fiber by thermally expanded core technique," Opt. Laser Technol., vol. 47, pp. 72-75, 2013.

[153] A. Kobyakov, M. Sauer, and D. Chowdhury, "Stimulated Brillouin scattering in optical fibers," Adv. Opt. Photon., vol. 2, no. 1, pp. 1-59, 2010.

[154] S. Smith, F. Zarinetchi, and S. Ezekiel, "Narrow-linewidth stimulated Brillouin fiber laser and applications," Opt. Lett., vol. 16, no. 6, pp. 393-395, 1991.

[155] J. Geng, S. Staines, Z. Wang, J. Zong, M. Blake, and S. Jiang, "Highly stable low-noise Brillouin fiber laser with ultranarrow spectral linewidth,” IEEE Photon. Technol. Lett., vol. 18, no. 17, pp. 1813-1815, 2006.

[156] M. Abbasnejad and A. Bijan, "FPGA-based implementation of a novel method for estimating the Brillouin frequency shift in BOTDA and BOTDR sensors," IEEE Sens. J., vol. 18, no. 5, pp. 2015-2022, 2017.

[157] D. lida and F. Ito, "Detection sensitivity of Brillouin scattering near Fresnel reflection in BOTDR measurement," J. Lightwave Technol., vol. 26, no. 4, pp. 417-424, 2008.

[158] G. Wang, L. Zhan, J. Liu, et al., "Watt-level ultrahigh-optical signal-to-noise ratio single-longitudinal-mode tunable Brillouin fiber laser," Opt. Lett., vol. 38, no. 1, pp. 19-21, 2013.

[159] J. Wang, Y. Hou, Q. Zhang, et al., "High-power, high signal-tonoise ratio single-frequency $1 \mu \mathrm{m}$ Brillouin all-fiber laser," Opt. Express, vol. 23, no. 22, pp. 28978-28984, 2015.

[160] G. J. Cowle and D. Yu. Stepanov, "Hybrid Brillouin/erbium fiber laser,” Opt. Lett., vol. 21, no. 16, pp. 1250-1252, 1996.

[161] X. Heng, J. Gan, Z. Zhang, et al., "Transverse mode switchable all-fiber Brillouin laser,” Opt. Lett., vol. 43, no. 17, pp. 4172-4175, 2018.

[162] N. Wang, J. C. Alvarado-Zacarias, M. S. Habib, H. Wen, Y. Zhang, J. E. Antonio-Lopez, P. Sillard, A. Amezcua-Correa, R. Amezcua-Correa, and G. Li, "High-order mode Brillouin fiber lasers based on intra- and inter-modal SBS," in Conference on Lasers and Electro-Optics, paper SM1L.1, 2019.

[163] J. Xu, L. Meng, L. Zhang, L. Teng, and X. Zeng, "Dynamic modeswitchable all-fiber Brillouin/Erbium laser," in Conference on Lasers and Electro-Optics, paper STh3P. 2, 2020.

[164] L. Feng, Y. Li, S. Wu, et al., "All-fiber generation of arbitrary cylindrical vector beams on the first-order Poincare sphere," Photon. Res., vol. 8, no. 8, pp. 1268-1277, 2020.

[165] J. Wang, "Twisted optical communications using orbital angular momentum," Sci. China Phys. Mech. Astron., vol. 62, no. 3, p. 034201, 2019.

[166] M. Gecevičius, R. Drevinskas, M. Beresna, and P. G. Kazansky, "Single beam optical vortex tweezers with tunable orbital angular momentum," Appl. Phys. Lett., vol. 104, no. 23, p. 231110, 2014.
[167] L. Yan, P. Gregg, E. Karimi, et al., “Q-plate enabled spectrally diverse orbital-angular momentum conversion for stimulated emission depletion microscopy," Optica, vol. 2, no. 10, pp. 900-903, 2015.

[168] R. Dorn, S. Quabis, and G. Leuchs, "Sharper focus for a radially polarized light beam," Phys. Rev. Lett., vol. 91, no. 23, p. 233901, 2003.

[169] K. Huang, J. Zeng, J. Gan, Q. Hao, and H. Zeng, "Controlled generation of ultrafast vector vortex beams from a mode-locked fiber laser," Opt. Lett., vol. 43, no. 16, pp. 3933-3936, 2018.

[170] W. Fu, L. G. Wright, P. Sidorenko, S. Backus, and F. W. Wise, "Several new directions for ultrafast fiber lasers [Invited]," Opt. Express, vol. 26, no. 8, pp. 9432-9463, 2018.

[171] W. H. Renninger and F. W. Wise, "Optical solitons in gradedindex multimode fibres," Nat. Commun., vol. 4, p. 1719, 2013.

[172] F. Wang, F. Shi, T. Wang, F. Pang, T. Wang, and X. Zeng, "Method of generating femtosecond cylindrical vector beams using broadband mode converter," IEEE Photon. Technol. Lett., vol. 29, no. 9, pp. 747-750, 2017.

[173] S. Huang, Y. Wang, P. Yan, J. Zhao, H. Li, and R. Lin, “Tunable and switchable multi-wavelength dissipative soliton generation in a graphene oxide mode-locked Yb-doped fiber laser," Opt. Express, vol. 22, no. 10, pp. 11417-11426, 2014.

[174] X. Hao, Z. Tong, W. Zhang, and Y. Cao, "A fiber laser temperature sensor based on SMF core-offset structure," Opt. Commun., vol. 335, pp. 78-81, 2015.

[175] N.-K. Chen, J.-W. Lin, F.-Z. Liu, and S.-K. Liaw, "Wavelengthtunable Er3+-doped fs mode-locked fiber laser using shortpass edge filters," IEEE Photon. Technol. Lett., vol. 22, no. 10, pp. 700-702, 2010.

[176] Y. W. Lee and B. Lee, "Wavelength-switchable erbium-doped fiber ring laser using spectral polarization dependent loss element," IEEE Photon. Technol. Lett., vol. 15, no. 6, pp. 795-797, 2003.

[177] Y. Qi, Z. Kang, J. Sun, et al., "Wavelength-switchable fiber laser based on few mode fiber filter with core-offset structure," Opt. Laser Technol., vol. 81, pp. 26-32, 2016.

[178] J. S. Milne, J. M. Dell, A. J. Keating, and L. Faraone, "Widely tunable MEMS-based Fabry-Perot filter," J. Microelectromech. Syst., vol. 18, no. 4, pp. 905-913, 2009.

[179] S. Yamashita, "Widely tunable erbium-doped fiber ring laser covering both c-band and l-band," IEEE J. Sel. Top. Quantum Electron., vol. 7, no. 1, pp. 41-43, 2001.

[180] J. Zhang, X. Qiao, F. Liu, et al., “A tunable erbium-doped fiber laser based on an $\mathrm{MZ}$ interferometer and a birefringence fiber filter," J. Opt., vol. 14, no. 1, p. 015402, 2012.

[181] Y. Li, L. Huang, S. Huang, et al., "Tunable narrow-linewidth fiber laser based on light-controlled graphene," J. Lightwave Technol., vol. 37, no. 4, pp. 1338-1344, 2019.

[182] X. He, Z. B. Liu, and D. N. Wang, "Wavelength-tunable, passively mode-locked fiber laser based on graphene and chirped fiber Bragg grating," Opt. Lett., vol. 37, no. 12, pp. 2394-2396, 2012.

[183] J. Wang, A. P. Zhang, Y. H. Shen, H. Y. Tam, and P. K. Wai, "Widely tunable mode-locked fiber laser using carbon nanotube and LPG W-shaped filter," Opt. Lett., vol. 40, no. 18, pp. 4329-4332, 2015.

[184] Z. X. Zhang, Z. W. Xu, and L. Zhang, "Tunable and switchable dual-wavelength dissipative soliton generation in an allnormal-dispersion $\mathrm{Yb}$-doped fiber laser with birefringence fiber filter," Opt. Express, vol. 20, no. 24, pp. 26736-26742, 2012. 
[185] H. Zhang, D. Tang, R. J. Knize, L. Zhao, Q. Bao, and K. P. Loh, "Graphene mode locked, wavelength-tunable, dissipative soliton fiber laser," Appl. Phys. Lett., vol. 96, no. 11, p. 111112, 2010.

[186] Q. Huang, C. Zou, C. Mou, et al., " $23 \mathrm{MHz}$ widely wavelengthtunable L-band dissipative soliton from an all-fiber Er-doped laser," Opt. Express, vol. 27, no. 14, pp. 20028-20036, 2019.

[187] L. Huang, W. Zhang, D. Mao, F. Gao, W. Peng, F. Bo, G. Zhang, and J. Xu, "All-fiber tunable ring laser based on an acoustooptic tunable coupler," in Conference on Lasers and ElectroOptics, paper JW2A.93, 2015.

[188] P. Chang, L. Huang, X. Song, F. Gao, G. Zhang, and J. Xu, "Acoustic wave induced Mach-Zehnder interferometer based on a sandwich-structured single mode fiber," in Conference on Lasers and Electro-Optics, paper JTu5A.88, 2016.

[189] P. Cheng, F. Shi, L. Meng, T. Wang, F. Pang, and X. Zeng, “Allfiber wavelength-tunable bandpass filter based on core-mode conversion for mode-locking laser," Opt. Eng., vol. 59, no. 9, p. 096101, 2020.

[190] R. Ismaeel, T. Lee, B. Oduro, Y. M. Jung, and G. Brambilla, “All-fiber fused directional coupler for highly efficient spatial mode conversion," Opt. Express, vol. 22, no. 10, pp. 11610-11619, 2014.

[191] H. Wan, J. Wang, Z. Zhang, Y. Cai, B. Sun, and L. Zhang, "High efficiency mode-locked, cylindrical vector beam fiber laser based on a mode selective coupler," Opt. Express, vol. 25, no. 10, pp. 11444-11451, 2017.

[192] A. W. Snyder, "Coupled-mode theory for optical fibers," J. Opt. Soc. Am., vol. 62, no. 62, pp. 1267-1277, 1972.

[193] T. A. Birks, P. S. J. Russell, and D. O. Culverhouse, "The acoustooptic effect in single-mode fiber tapers and couplers," J. Lightwave Technol., vol. 14, no. 11, pp. 2519-2529, 1996.

[194] Y. Wang, F. Leo, J. Fatome, M. Erkintalo, S. G. Murdoch, and S. Coen, "Universal mechanism for the binding of temporal cavity solitons," Optica, vol. 4, no. 8, pp. 855-863, 2017.

[195] Z. Qin, G. Xie, H. Gu, et al., "Mode-locked 2.8- $\mu$ m fluoride fiber laser: from soliton to breathing pulse," Adv. Photon., vol. 1, no. 6, p. 065001, 2019.

[196] X. Liu, X. Yao, and Y. Cui, "Real-time observation of the buildup of soliton molecules," Phys. Rev. Lett., vol. 121, no. 2, p. 023905, 2018.

[197] B. Li, S. W. Huang, Y. Li, C. W. Wong, and K. K. Y. Wong, "Panoramic-reconstruction temporal imaging for seamless measurements of slowly-evolved femtosecond pulse dynamics," Nat. Commun., vol. 8, no. 1, p. 61, 2017.

[198] E. J. R. Kelleher and J. C. Travers, "Chirped pulse formation dynamics in ultra-long mode-locked fiber lasers," Opt. Lett., vol. 39, no. 6, pp. 1398-1401, 2014.

[199] H. Chen, M. Liu, J. Yao, et al., "Buildup dynamics of dissipative soliton in an ultrafast fiber laser with net-normal dispersion," Opt. Express, vol. 26, no. 3, pp. 2972-2982, 2018.

[200] H. Chen, M. Liu, J. Yao, et al., "Soliton booting dynamics in an ultrafast anomalous dispersion fiber laser," IEEE Photon. J., vol. 10, no. 2, p. 1501809, 2018.

[201] A. Chong, L. Wright, and F. Wise, "Ultrafast fiber lasers based on self-similar pulse evolution: a review of current progress," Rep. Prog. Phys., vol. 78, no. 11, p. 113901, 2015.
[202] G. Herink, B. Jalali, C. Ropers, and D. R. Solli, "Resolving the build-up of femtosecond mode-locking with single-shot spectroscopy at $90 \mathrm{MHz}$ frame rate," Nat. Photonics, vol. 10, pp. 321-326, 2016.

[203] K. Goda, K. K. Tsia, and B. Jalali, "Serial time-encoded amplified imaging for real-time observation of fast dynamic phenomena," Nature, vol. 458, pp. 1145-1149, 2009.

[204] A. Mahjoubfar, D. V. Churkin, S. Barland, N. Broderick, S. K. Turitsyn, and B. Jalali, "Time stretch and its applications," Nat. Photonics, vol. 11, no. 6, pp. 341-351, 2017.

[205] Y. Xu, X. Wei, Z. Ren, K. K. Y. Wong, and K. K. Tsia, “Ultrafast measurements of optical spectral coherence by single-shot time-stretch interferometry," Sci. Rep., vol. 6, p. 27937, 2016.

[206] A. van de Walle, M. Hanna, F. Guichard, et al., "Spectral and spatial full-bandwidth correlation analysis of bulk-generated supercontinuum in the mid-infrared," Opt. Lett., vol. 40, no. 4, pp. 673-676, 2015

[207] D. Solli, C. Ropers, P. Koonath, and B. Jalali, "Optical rogue waves," Nature, vol. 450, pp. 1054-1057, 2007.

[208] X. Liu and Y. Cui, "Revealing the behavior of soliton buildup in a mode-locked laser," Adv. Photon., vol. 1, no. 1, p. 016003, 2019.

[209] Y. Cui and X. Liu, "Revelation of the birth and extinction dynamics of solitons in SWNT-mode-locked fiber lasers," Photon. Res., vol. 7, no. 4, pp. 53-60, 2019.

[210] X. Liu and M. Pang, "Revealing the buildup dynamics of harmonic mode-locking states in ultrafast lasers," Laser Photon. Rev., vol. 13, no. 9, p. 1970038, 2019.

[211] X. Liu, D. Popa, and N. Akhmediev, "Revealing the transition dynamics from Q switching to mode locking in a soliton laser," Phys. Rev. Lett., vol. 123, p. 093901, 2019.

[212] P. Ryczkowski, M. Närhi, C. Billet, J.-M. Merolla, G. Genty, and J. M. Dudley, "Real-time full-field characterization of transient dissipative soliton dynamics in a mode-locked laser," Nat. Photonics, vol. 12, pp. 221-227, 2018.

[213] J. Peng and H. Zeng, "Build-up of dissipative optical soliton molecules via diverse soliton interactions," Laser Photon. Rev., vol. 12, no. 8, p. 1800009, 2018.

[214] I. Kudelin, S. Sugavanam, and M. Chernysheva, "Build-up dynamics in bidirectional soliton fiber lasers," Photon. Res., vol. 8, no. 6, pp. 776-780, 2020.

[215] L. G. Wright, D. N. Christodoulides, and F. W. Wise, "Spatiotemporal mode-locking in multimode fiber lasers," Science, vol. 358, no. 6359, pp. 94-97, 2017.

[216] L. G. Wright, Z. M. Ziegler, P. M. Lushnikov, et al., “Multimode nonlinear fiber optics: massively parallel numerical solver, tutorial, and outlook," IEEE J. Sel. Top. Quantum Electron., vol. 24, no. 3, p. 5100516, 2018.

[217] L. G. Wright, D. N. Christodoulides, and F. W. Wise, "Controllable spatiotemporal nonlinear effects in multimode fibres," Nat. Photonics, vol. 9, no. 5, pp. 306-310, 2015.

[218] A. F. J. Runge, N. G. R. Broderick, and M. Erkintalo, "Observation of soliton explosions in a passively mode-locked fiber laser," Optica, vol. 2, no. 1, pp. 36-39, 2015.

[219] Y. Luo, R. Xia, P. P. Shum, et al., "Real-time dynamics of soliton triplets in fiber lasers," Photon. Res., vol. 8, no. 6, pp. 884-891, 2020. 